\title{
UNIVALENT HARMONIC FUNCTIONS
}

\author{
W. HENGARTNER AND G. SCHOBER
}

\begin{abstract}
Several families of complex-valued, univalent, harmonic functions are studied from the point of view of geometric function theory. One class consists of mappings of a simply-connected domain onto an infinite horizontal strip with a normalization at the origin. Extreme points and support points are determined, as well as sharp estimates for Fourier coefficients and distortion theorems. Next, mappings in $|z|>1$ are considered that leave infinity fixed. Some coefficient estimates, distortion theorems, and covering properties are obtained. For such mappings with real boundary values, many extremal problems are solved explicitly.
\end{abstract}

1. Introduction. Recently, J. Clunie and T. Sheil-Small [2] studied the class $S_{H}$ of all harmonic, complex-valued, orientation-preserving, univalent mappings $f$ defined on the open unit disk $U$, which are normalized at the origin by $f(0)=0$ and $f_{z}(0)=1$. Such functions admit the representation

$$
f=h+\bar{g}
$$

where

$$
h(z)=z+\sum_{k=2}^{\infty} a_{k} z^{k} \quad \text { and } \quad g(z)=\sum_{k=1}^{\infty} b_{k} z^{k}
$$

are analytic in $U$.

One shows easily that the orientation-preserving property implies that $\left|b_{1}\right|<1$. Therefore $\left(f-\overline{b_{1} f}\right) /\left(1-\left|b_{1}\right|^{2}\right)$ is also in $S_{H}$ and one may restrict attention to the subclass

$$
S_{H}^{0}=\left\{f \in S_{H} \text { normalized by } f(0)=0, f_{z}(0)=1 \text {, and } f_{\bar{z}}(0)=0\right\} .
$$

From [2] we have the important facts that $S_{H}$ is normal and that $S_{H}^{0}$ is compact with respect to the topology of locally uniform convergence. In addition, let us mention the following interesting result from [2].

LEMMA 1.1 [2, THEOREM 5.7]. A function $f=h+\bar{g}$ in $S_{H}$ maps $U$ onto a convex domain if and only if the analytic function $h-e^{2 i \theta} g$ is univalent and maps $U$ onto a domain convex in the direction $\theta$ for all $\theta, 0 \leq \theta<\pi$.

In contrast to conformal mappings, harmonic mappings are not at all determined (up to normalization) by their image domains. Therefore, it is natural to study

Received by the editors January 21, 1985.

1980 Mathematics Subject Classification (1985 Revision). Primary 30C55, 58E20; Secondary 30C75, 31A10, 42A16.

Key words and phrases. Harmonic mappings, extremal problems.

This work was supported in part by grants from the National Research Council (Canada) and the National Science Foundation (U.S.). 
the class $S_{H}(D, G)$ of harmonic orientation-preserving univalent mappings of one domain $D$ onto another domain $G$. We shall assume that both $D$ and $G$ contain the origin and that functions $f \in S_{H}(D, G)$ are normalized so that

$$
f(0)=0, \quad f_{z}(0)>0, \quad \text { and } \quad f_{\bar{z}}(0)=0 .
$$

If $\psi$ is a conformal mapping of a domain $D_{1}$ onto $D_{2}$ such that $\psi(0)=0$ and $\psi^{\prime}(0)>0$, note that

$$
S_{H}\left(D_{1}, G\right)=S_{H}\left(D_{2}, G\right) \circ \psi
$$

For the special case $D=G=U$ we refer to the work of G. Choquet [1], E. Heinz [4], and R. R. Hall [3].

In the next section of this article we choose the domain $G$ to be a strip $\Omega$ instead of a disk and the domain $D \neq \mathbf{C}$ to be simply connected. Since $S_{H}(D, \Omega)$ is not closed, we determine the extreme points and support points of its closure $\overline{S_{H}(D, \Omega)}$. We are able to give an isomorphism between $\overline{S_{H}(D, \Omega)}$ and the familiar class $P$ of analytic functions $f$ in $U$ with positive real part and $f(0)=1$. As applications we give some coefficient estimates and distortion theorems. In particular, we refer to Theorem 2.14.

In the third section we consider harmonic orientation-preserving univalent mappings defined on $\widetilde{U}=\{z:|z|>1\}$ that map $\infty$ to $\infty$. Such mappings can be represented by

$$
f(z)=A \log |z|+h(z)+\overline{g(z)}
$$

where

$$
h(z)=\alpha z+\sum_{k=0}^{\infty} a_{k} z^{-k} \quad \text { and } \quad g(z)=\beta z+\sum_{k=1}^{\infty} b_{k} z^{-k}
$$

are analytic in $\widetilde{U}$ and $|\alpha|>|\beta|$. Since the affine transformation

$$
\left(\bar{\alpha} f-\overline{\beta f}-\bar{\alpha} a_{0}+\overline{\beta a_{0}}\right) /\left(|\alpha|^{2}-|\beta|^{2}\right)
$$

is again in the class, we may restrict our attention to the family $\Sigma_{H}^{\prime}$ of all harmonic orientation-preserving univalent mappings which have the development

$$
f(z)=z+A \log |z|+\sum_{k=1}^{\infty} a_{k} z^{-k}+\overline{\sum_{k=1}^{\infty} b_{k} z^{-k}}
$$

We show that $\Sigma_{H}^{\prime}$ is compact, and by Schwarz's lemma it follows that $|A| \leq 2$ and $\left|b_{1}\right| \leq 1$. In contrast to the familiar analytic subclass $\Sigma^{\prime}$, but similar to the class $S_{H}^{0}$, support points of $\Sigma_{H}^{\prime}$ need not be slit mappings. In particular, for real $\gamma$ the function $z+e^{i \gamma} / \bar{z}$ is a support point of $\Sigma_{H}^{\prime}$ and maps $\widetilde{U}$ onto $\{w:|w|>2 \cos (\gamma / 2)\}$. In addition, since the image domain is $\mathbf{C} \backslash\{0\}$ when $\gamma=\pi$, we note that harmonic mappings need not preserve the conformal type of a domain. $\S 3$ closes with sharp lower bounds for the diameter of $\mathbf{C} \backslash f(\widetilde{U})$ as a function of $b_{1}$ and with an area theorem.

Finally, in $\S 4$ we study the subclass $\Sigma_{H \mathbf{R}}^{\prime}$ of all functions in $\Sigma_{H}^{\prime}$ that map $\widetilde{U}$ onto the complement of a real line segment. We show that functions in $\Sigma_{H \mathbf{R}}^{\prime}$ can be represented, but in a nonlinear fashion, in terms of probability measures on the unit circle. Applications include sharp coefficient estimates and distortion theorems, including the maximum diameter of the omitted segment. 
2. Harmonic mappings onto a strip. We shall use the strip

$$
\Omega=\{w:|\operatorname{Im} w|<\pi / 4\}
$$

and a simply-connected domain $D \neq \mathbf{C}$ containing the origin. Then $S_{H}(D, \Omega)$ consists of harmonic orientation-preserving univalent mappings $f=u+i v$ from $D$ onto $\Omega$ normalized by

$$
u(0)=v(0)=0, \quad u_{y}(0)=v_{x}(0)=0, \quad \text { and } \quad u_{x}(0)=v_{y}(0)>0 .
$$

In addition, we may represent $f=h+\bar{g}$ where $h$ and $g$ are analytic in $D$ and have the expansions $h(z)=\sum_{k=1}^{\infty} a_{k} z^{k}$ with $a_{1}>0$ and $g(z)=\sum_{k=2}^{\infty} b_{k} z^{k}$ in a neighborhood of the origin. Since $f$ preserves orientation, the function $a(z)=$ $g^{\prime}(z) / h^{\prime}(z)$ satisfies $|a(z)|<1$, and the normalization implies $a(0)=0$.

In this section $\psi_{D}$ denotes the conformal mapping from $D$ onto the unit disk normalized by $\psi_{D}(0)=0$ and $\psi_{D}^{\prime}(0)>0$. Since $S_{H}(D, \Omega)=S_{H}(U, \Omega) \circ \psi_{D}$, it is sufficient for many problems to consider the class $S_{H}(U, \Omega)$. Another particularly interesting case is $D=\Omega$. Then $S_{H}(\Omega, \Omega)$ consists of automorphisms of $\Omega$.

The following lemmas prepare for an integral representation of functions in $S_{H}(U, \Omega)$.

Lemma 2.1. Consider the Dirichlet problem for $\Delta v=0$ in $U$ with boundary values $v=\pi / 4$ on an open arc $\Gamma$ of $\partial U$ and $v=-\pi / 4$ on the complementary arc, i.e., on $\partial U \backslash \bar{\Gamma}$. Then the only bounded solution with the normalizations $v(0)=0$, $v_{x}(0)=0$, and $v_{y}(0)>0$ is

$$
\Gamma=\left\{e^{i t}: 0<t<\pi\right\} \quad \text { and } \quad v(z)=\frac{1}{2} \arg \left(\frac{1+z}{1-z}\right) .
$$

Proof. Let $\Gamma=\left\{e^{i t}: t_{1}<t<t_{2}\right\}$. Then the bounded solution of the Dirichlet problem is

$$
v(z)=-\frac{\pi}{4}+\frac{1}{4} \int_{t_{1}}^{t_{2}} \operatorname{Re} \frac{e^{i t}+z}{e^{i t}-z} d t .
$$

The condition $v(0)=0$ implies $t_{2}=t_{1}+\pi$, and since

$$
v_{x}(0)+i v_{y}(0)=\frac{1}{2} \int_{t_{1}}^{t_{1}+\pi} e^{i t} d t=i e^{i t_{1}},
$$

the remaining normalization forces $t_{1}=0$ (modulo $2 \pi$ ). Thus $\Gamma$ and also $v$ are as asserted.

For $z \in U$ and $|\eta|=1$, define now the kernel

$$
\begin{aligned}
K(z, \eta) & =\int_{0}^{z} \frac{1+\eta z}{1-\eta z} \frac{d z}{1-z^{2}} \\
& = \begin{cases}z /(1-z) & \text { if } \eta=1 \\
z /(1+z) & \text { if } \eta=-1, \\
\frac{1}{2}\left(\frac{1+\eta}{1-\eta}\right) & \log \left(\frac{1-\eta z}{1-z}\right)-\frac{1}{2}\left(\frac{1-\eta}{1+\eta}\right) \log \left(\frac{1-\eta z}{1+z}\right) \quad \text { if } \eta \neq \pm 1 .\end{cases}
\end{aligned}
$$


Then for $\eta=e^{-2 i \alpha}$ we define

$$
\begin{aligned}
u_{\alpha}(z)= & \operatorname{Re} K\left(z, e^{-2 i \alpha}\right) \\
= & \begin{cases}\operatorname{Re}\{z /(1-z)\} & \text { if } \alpha=0, \\
\operatorname{Re}\{z /(1+z)\} & \text { if } \alpha=\pi / 2, \\
\frac{1}{2}(\cot \alpha) \arg \left(\frac{1-e^{-2 i \alpha} z}{1-z}\right)+\frac{1}{2}(\tan \alpha) \arg \left(\frac{1-e^{-2 i \alpha} z}{1+z}\right) & \text { if } 0<|\alpha|<\frac{\pi}{2},\end{cases}
\end{aligned}
$$

as well as the family

$$
\mathcal{F}=\left\{f: f(z)=\operatorname{Re} \int_{|\eta|=1} K(z, \eta) d \mu(\eta)+\frac{i}{2} \arg \left(\frac{1+z}{1-z}\right), \mu \in \mathcal{P}\right\}
$$

where $P$ is the set of probability measures on the Borel sets of $|\eta|=1$.

LEMMA 2.2. We have $S_{H}(U, \Omega) \subset \mathcal{F}$.

Proof. Let $f \in S_{H}(U, \Omega)$. Decompose $f=h+\bar{g}$ as before so that $a=g^{\prime} / h^{\prime}$ satisfies the hypothesis of Schwarz's lemma. Note that $u=\operatorname{Re} f=\operatorname{Re}\{h+g\}$ and $v=\operatorname{Im} f=\operatorname{Im}\{h-g\}$. The function $v=\operatorname{Im} f$ is harmonic and bounded in $U$, and it has the normalizations of Lemma 2.1. Its boundary values are $\pi / 4$ and $-\pi / 4$ on complementary $\operatorname{arcs}$ of $\partial U$. Therefore Lemma 2.1 implies $v(z)=$ $\frac{1}{2} \arg ((1+z) /(1-z))$ and

$$
h^{\prime}(z)-g^{\prime}(z)=2 i v_{z}(z)=\frac{1}{1-z^{2}}
$$

At the same time,

$$
h^{\prime}(z)+g^{\prime}(z)=\left[h^{\prime}(z)-g^{\prime}(z)\right] \frac{1+a(z)}{1-a(z)}=\frac{1}{1-z^{2}} p(z)
$$

where by the Herglotz formula $p(z)=\int_{|\eta|=1}(1+\eta z) /(1-\eta z) d \mu$ for some measure $\mu \in \mathcal{P}$. Therefore

$$
u(z)=\operatorname{Re}\left\{\int_{0}^{z}\left[h^{\prime}(z)+g^{\prime}(z)\right] d z\right\}=\operatorname{Re}\left\{\int_{0}^{z} \frac{p(z) d z}{1-z^{2}}\right\}=\operatorname{Re}\left\{\int_{|\eta|=1} K(z, \eta) d \mu\right\}
$$

and the lemma is proved.

As a first consequence we obtain the folowing interesting result.

THEOREM 2.3. Let $f=u+i v \in S_{H}(D, \Omega)$. Then $u_{x}(0)=v_{y}(0)=\psi_{D}^{\prime}(0)$ is independent of $f$. In other words, we have

$$
f(z)=\psi_{D}^{\prime}(0) z+\sum_{k=2}^{\infty} a_{k} z^{k}+\overline{\sum_{k=2}^{\infty} b_{k} z^{k}}
$$

for all $f \in S_{H}(D, \Omega)$. In particular, if $D=U$ or $D=\Omega$, then the leading coefficient is 1 .

Proof. Each $f \in \mathcal{F}$ had leading coefficient $f_{z}(0)=1$, and so each $f \in$ $S_{H}(D, \Omega)=S_{H}(U, \Omega) \circ \psi_{D} \subset \exists \circ \psi_{D}$ has leading coefficient $f_{z}(0)=\psi_{D}^{\prime}(0)$. 
REMARK. Theorem 2.3 depends strongly on the choice of image domain $\Omega$. Indeed, it fails for example for $S_{H}(D, U)$. In fact, Hall [3] has shown for $f(z)=$ $\sum_{k=1}^{\infty} a_{k} z^{k}+\sum_{k=2}^{\infty} b_{k} z^{k} \in S_{H}(U, U)$ that

$$
\frac{3 \sqrt{3}}{2 \pi} \leq a_{1} \leq 1
$$

where the bounds are best possible.

The following lemma gives properties of the family $\mathcal{F}$.

LEMMA 2.4. F is a compact convex set of normalized univalent orientationpreserving harmonic mappings from $U$ into $\Omega$.

Proof. It is evident from the definition (2.2) that $₹$ is a compact convex set of normalized harmonic functions in $U$. If $f \in \mathcal{F}$, then $\operatorname{Im} f=\operatorname{Im} \psi_{\Omega}^{-1}$ where $\psi_{\Omega}^{-1}(z)=\frac{1}{2} \log ((1+z) /(1-z))$ maps $U$ conformally onto $\Omega$. Therefore $f \circ \psi_{\Omega}$ maps horizontal lines in $\Omega$ into themselves. In addition, if $\sigma+i \tau \in \Omega$, then $(\partial f / \partial \tau) \circ \psi_{\Omega}=$ $\operatorname{Re} p \circ \psi_{\Omega}$ for some function $p$ of positive real part. Therefore $f$ is univalent and orientation-preserving and maps $\Omega$ into itself.

REMARKS. (1) There are harmonic mappings from $U$ into $\Omega$ which are not in ₹. Indeed, no conformal mapping of $U$ onto a proper subdomain of $\Omega$ can have the normalization of $₹$.

(2) $S_{H}(U, \Omega) \neq \mathcal{F}$. For instance, if $\mu$ is a unit point mass at $\eta=1$, then

$$
f(z)=\operatorname{Re}\left\{\frac{z}{1-z}\right\}+\frac{1}{2} \arg \left(\frac{1+z}{1-z}\right)
$$

maps $U$ onto the half-strip $\left\{w: \operatorname{Re} w>-\frac{1}{2},|\operatorname{Im} w|<\pi / 4\right\}$. Therefore $f \in$ $\mathcal{F} \backslash S_{H}(U, \Omega)$.

Although functions in $₹$ do not necessarily map onto $\Omega$, we shall see that they map onto convex subdomains. Later, in Theorem 2.9, we shall be even more specific.

LEMMA 2.5. If $f \in \mathcal{F}$, then $f(U)$ is convex.

Proof. If $f=h+\bar{g} \in \mathcal{F}$, then by Lemmas 1.1 and 2.4 the set $f(U)$ will be convex if and only if the analytic functions $h-e^{2 i \theta} g$ are univalent and convex in the direction $\theta$ for all $\theta, 0 \leq \theta<\pi$. To show the latter, it is sufficient to show that the functions $F=i e^{-i \theta}\left(h-e^{2 i \theta} g\right)$ are univalent and convex in the vertical direction. By [5, Theorem 1] this will be the case if $\operatorname{Re}\left\{\left(1-z^{2}\right) F^{\prime}(z)\right\}>0$. Thus we verify that

$$
\begin{aligned}
\operatorname{Re}\left\{\left(1-z^{2}\right) F^{\prime}(z)\right\} & =-\operatorname{Im}\left\{e^{-i \theta}\left(1-z^{2}\right)\left[h^{\prime}(z)-e^{2 i \theta} g^{\prime}(z)\right]\right\} \\
& =(\sin \theta) \operatorname{Re}\left\{\int_{|\eta|=1} \frac{1+\eta z}{1-\eta z} d \mu\right\}
\end{aligned}
$$

is positive for $0<\theta<\pi$. For $\theta=0$ the function $h-g=\frac{1}{2} \log ((1+z) /(1-z))$ is univalent and convex.

The next lemma gives a sufficient condition in order that $f \in \mathcal{F}$ implies $f \in$ $S_{H}(U, \Omega)$. Denote $\|a\|_{\infty}=\sup _{z \in U}|a(z)|$.

LEMMA 2.6. If $f=h+\bar{g} \in \mathcal{F}$ and $\left\|g^{\prime} / h^{\prime}\right\|_{\infty}<1$, then $f(U)=\Omega$ and $f \in$ $S_{H}(U, \Omega)$.

Proof. By Lemma 2.4 and the hypothesis that $\left\|f_{\bar{z}} / f_{z}\right\|_{\infty}=\left\|g^{\prime} / h^{\prime}\right\|_{\infty}<1, f$ is a quasiconformal mapping of $U$ into $\Omega$. By Lemma 2.5, $f(U)$ is convex and if it were 
not all of $\Omega$, then $\partial f(U)$ would be a Jordan curve in $\mathbf{C} \cup\{\infty\}$. Consequently, the quasiconformal mapping $f$ would extend [6, Theorem I.8.2] to a homeomorphism (in the spherical metric) between $\bar{U}$ and $\overline{f(U)}$. However, Lemma 2.1 implies that $f$ has a discontinuity at either $z=1$ or $z=-1$. Thus $f(U)=\Omega$ and $f \in S_{H}(U, \Omega)$.

Finally, we are able to identify $\mathcal{F}$ as the closure of $S_{H}(U, \Omega)$.

THEOREM 2.7. $\overline{S_{H}(U, \Omega)}=\mathcal{F}$ and $\overline{S_{H}(D, \Omega)}=\xi \circ \psi_{D}$.

Proof. $S_{H}(U, \Omega) \subset \mathcal{F}$ by Lemma 2.2. At the same time, each $f \in \mathcal{F}$ has a measure $\mu \in \mathcal{P}$ which can be approximated by measures $\mu_{n} \in \mathcal{P}$ so that the corresponding functions $f_{n}=h_{n}+\overline{g_{n}} \in \mathcal{F}$ have $\left\|g_{n}^{\prime} / h_{n}^{\prime}\right\|_{\infty}=\left\|\left(p_{n}-1\right) /\left(p_{n}+1\right)\right\|_{\infty}<$ 1. By Lemma 2.6 the functions $f_{n}$ belong to $S_{H}(U, \Omega)$ and so $\overline{S_{H}(U, \Omega)}=\mathcal{F}$ follows. The final assertion is obvious.

Theorem 2.7 is very useful for solving extremal problems on $S_{H}(D, \Omega)$. In particular, if $\phi$ is a real continuous convex functional on $\overline{S_{H}(D, \Omega)}$, it is sufficient by the Krein-Milman theorem to find the maximum of $\phi$ over the set of extreme points.

THEOREM 2.8. The set of extreme points of $\overline{S_{H}(U, \Omega)}$ is the set of functions

$$
f_{\alpha}(z)=u_{\alpha}(z)+\frac{i}{2} \arg \left(\frac{1+z}{1-z}\right), \quad-\frac{\pi}{2}<\alpha \leq \frac{\pi}{2},
$$

where $u_{\alpha}$ is defined in (2.1). Furthermore, the image $f_{\alpha}(U)$ is

(a) the half-strip $\left\{w \in \Omega: \operatorname{Re} w>-\frac{1}{2}\right\}$ if $\alpha=0$,

(b) the half-strip $\left\{w \in \Omega: \operatorname{Re} w<\frac{1}{2}\right\}$ if $\alpha=\pi / 2$,

(c) the interior of the triangle with vertices

$$
\begin{aligned}
& V_{1}=\frac{1}{2}\left[\left(\frac{\pi}{2}-\alpha\right) \tan \alpha+(\pi-\alpha) \cot \alpha\right]+\frac{i \pi}{4}, \\
& V_{2}=-\frac{1}{2}\left[\left(\frac{\pi}{2}+\alpha\right) \tan \alpha+\alpha \cot \alpha\right]+\frac{i \pi}{4}, \quad \text { and } \\
& V_{3}=\frac{1}{2}\left[\left(\frac{\pi}{2}-\alpha\right) \tan \alpha-\alpha \cot \alpha\right]-\frac{i \pi}{4} \quad \text { if } 0<\alpha<\frac{\pi}{2}, \quad \text { and }
\end{aligned}
$$

(d) the interior of the triangle with vertices

$$
\begin{aligned}
& V_{1}=-\frac{1}{2}\left[\left(\frac{\pi}{2}+\alpha\right) \tan \alpha+(\pi+\alpha) \cot \alpha\right]-\frac{i \pi}{4}, \\
& V_{2}=\frac{1}{2}\left[\left(\frac{\pi}{2}-\alpha\right) \tan \alpha-\alpha \cot \alpha\right]-\frac{i \pi}{4}, \quad \text { and } \\
& V_{3}=-\frac{1}{2}\left[\left(\frac{\pi}{2}+\alpha\right) \tan \alpha+\alpha \cot \alpha\right]+\frac{i \pi}{4} \quad \text { for }-\frac{\pi}{2}<\alpha<0 .
\end{aligned}
$$

ProOF. Since $\overline{S_{H}(U, \Omega)}=\mathcal{F}$, one can easily identify the extreme points from the integral representation in (2.2). In particular, the mapping

$$
\mu \in P \rightarrow \operatorname{Re} \int_{|\eta|=1} K(\cdot, \eta) d \mu
$$

is a linear homeomorphism, and we know that the extreme points of $\mathcal{P}$ are unit point masses. Thus the extreme points are as indicated. 
Since the mappings are very elementary, it is clear that $f_{0}(U)$ and $f_{\pi / 2}(U)$ are the half-strips (a) and (b). Next, let $0<\alpha<\pi / 2$ and define

$$
\begin{aligned}
& \omega_{1}(z)=\frac{\alpha}{\pi}+\frac{1}{\pi} \arg \left(\frac{1-e^{-2 i \alpha} z}{1-z}\right), \\
& \omega_{2}(z)=\frac{1}{2}-\frac{\alpha}{\pi}-\frac{1}{\pi} \arg \left(\frac{1-e^{-2 i \alpha} z}{1+z}\right), \quad \text { and } \\
& \omega_{3}(z)=1-\omega_{1}(z)-\omega_{2}(z)=\frac{1}{2}-\frac{1}{\pi} \arg \left(\frac{1+z}{1-z}\right)
\end{aligned}
$$

for $z \in U$. Then $\omega_{1}$ is the harmonic measure of the arc $\left\{e^{i \theta}: 0<\theta<2 \alpha\right\}, \omega_{2}$ is the harmonic measure of the $\operatorname{arc}\left\{e^{i \theta}: 2 \alpha<\theta<\pi\right\}$, and $\omega_{3}$ is the harmonic measure of the remaining arc $\left\{e^{i \theta}:-\pi<\theta<0\right\}$. It is straightforward to verify that

$$
f_{\alpha}=V_{1} \omega_{1}+V_{2} \omega_{2}+V_{3} \omega_{3}
$$

where $V_{1}, V_{2}$, and $V_{3}$ are the points in (c). Thus $f_{\alpha}(U)$ consists of all proper convex combinations of these three points, and so (c) is verified.

Since $f_{\alpha}(z)=\overline{f_{-\alpha}(\bar{z})}$, the case (d) follows from (c) by conjugating and replacing $\alpha$ by $-\alpha$.

REMARKS. (1) The formula (2.4) is a nice potential-theoretic representation for the functions $f_{\alpha}$. Let us give a more geometric one. Let $0<\alpha<\pi / 2$ and define $\theta(z)$ to be the angle at $z$ generated by the rays from $z$ to 1 and from $z$ to $e^{2 i \alpha}$. Then

$$
u_{\alpha}(z)=\frac{\theta(z)-2 \alpha}{\sin 2 \alpha}+\frac{\pi}{2}(\tan \alpha)\left[\omega_{3}(z)-\frac{1}{2}\right],
$$

where $\omega_{3}$ is the harmonic measure of the lower semicircle, defined earlier.

(2) For $0<\alpha<\pi / 2$ we have $u_{(\pi / 2)-\alpha}(z)=-u_{\alpha}(-\bar{z})$.

(3) The extreme points of $\overline{S_{H}(D, \Omega)}$ are the functions $f_{\alpha} \circ \psi_{D}$ for $-\pi / 2<\alpha \leq$ $\pi / 2$.

(4) No extreme point of $\overline{S_{H}(D, \Omega)}$ belongs to $S_{H}(D, \Omega)$ ! This follows by observing that each extreme point maps to a proper subset of $\Omega$.

The next theorem describes the functions in $\overline{S_{H}(D, \Omega)} \backslash S_{H}(D, \Omega)$.

THEOREM 2.9. Let $f \in \overline{S_{H}(D, \Omega)}$. (a) If $\operatorname{Re} f$ is bounded above, then $f(D)$ is bounded to the right by a straight line segment.

(b) If $\operatorname{Re} f$ is bounded below, then $f(D)$ is bounded to the left by a straight line segment.

(c) If $\operatorname{Re} f$ is bounded neither from below nor from above, then $f(D)=\Omega$ and $f \in S_{H}(D, \Omega)$.

ProOF. With no loss of generality we may assume $D=U$. If $f \in \overline{S_{H}(U, \Omega)}$, then Theorem 2.7 and Lemma 2.5 imply that $f(U)$ is a convex subset of $\Omega$. Recall also that $f \circ \psi_{\Omega}$ maps horizontal lines into themselves. Therefore assertion (c) is obvious.

To prove (a) we want to show that $\lim _{x \rightarrow \infty} u \circ \psi_{\Omega}(x+i y)=u^{*}(y)$ is linear for $|y|<$ $\pi / 4$. On the boundary of the subdomain $\Omega_{0}=\left\{x+i y: x>0,|y|<y_{0}\right\}, y_{0}<\pi / 4$, the function $u \circ \psi_{\Omega}$ is continuous except possibly for a jump discontinuity at $\infty$ of amount $\delta=u^{*}\left(y_{0}\right)-u^{*}\left(-y_{0}\right)$. Since the harmonic function $u \circ \psi_{\Omega}(x+i y)-\frac{1}{2}\left(\delta / y_{0}\right) y$ 
is continuous on $\partial \Omega_{0}$ even at $\infty$, the linearity of $u^{*}(y)$ follows, at least for $|y|<y_{0}$. Since $y_{0}$ is arbitrary, (a) is proved.

The statement (b) follows by applying (a) to $-f(-z)$.

The set $H(D)$ of complex-valued harmonic functions in $D$ with the topology of locally uniform convergence forms a locally convex topological vector space. Its topological dual space $H^{\prime}(D)$ can be represented, for example, by complex measures with compact support in $D$. A support point of a set $K \subset H(D)$ is a function $f_{0} \in K$ such that for some $L \in H^{\prime}(D)$ with $\operatorname{Re} L$ nonconstant on $K$ we have

$$
\operatorname{Re} L\left(f_{0}\right) \geq \operatorname{Re} L(f) \text { for all } f \in K \text {. }
$$

Our next theorem describes the support points of $\overline{S_{H}(D, \Omega)}$.

THEOREM 2.10. The set of support points of $\overline{S_{H}(D, \Omega)}$ is the set of all finite convex combinations of extreme points of $\overline{S_{H}(D, \Omega)}$, that is, the set of functions of the form $\sum_{k=1}^{N} \lambda_{k}\left(f_{\alpha_{k}} \circ \psi_{D}\right)$ where $\lambda_{k}>0, \sum_{k=1}^{N} \lambda_{k}=1$, and $f_{\alpha_{k}}$ is defined in (2.3).

ProOF. Again, with no loss of generality we may assume $D=U$. Corresponding to $L \in H^{\prime}(U)$ we first define a real-linear function $l$ on the analytic subspace of $H(U)$ by

$$
l\left(\frac{\partial}{\partial z} \operatorname{Re} f\right)=\operatorname{Re}\{L(f)-L(1) f(0)\} .
$$

Then $\widetilde{L}(\phi)=l(\phi)-i l(i \phi)$ defines a continuous complex-linear functional $\widetilde{L}$ on the analytic subspace of $H(U)$. If $f=u+i v$ belongs to $\overline{S_{H}(U, \Omega)}=\mathcal{F}$, then

$$
\operatorname{Re} L(f)=l\left(u_{z}\right)=l\left(\frac{p(z)}{1-z^{2}}\right)=\operatorname{Re} \widetilde{L}\left(\frac{p(z)}{1-z^{2}}\right)
$$

for a corresponding function $p \in P$, the family of analytic functions in $U$ with positive real part and $p(0)=1$. In other words, $f_{0}$ is optimal for $\operatorname{Re} L$ over $\overline{S_{H}(U, \Omega)}$ if and only if the corresponding $p_{0}$ is optimal for $\operatorname{Re} \widetilde{L}\left(p(z) /\left(1-z^{2}\right)\right)$ over $P$. The support points of $P$ are known (see, e.g., [8, Theorem 1]) to be generated by finitely discrete measures $\mu \in P$. Thus support points of $\overline{S_{H}(U, \Omega)}$ have the indicated form. Since our construction is reversible, the indicated functions are all support points.

COROLlaRY 2.11. A function $f=h+\bar{g}$ in $\overline{S_{H}(U, \Omega)}$ is a support point if and only if $a=g^{\prime} / h^{\prime}$ is a finite Blaschke product and $a(0)=0$.

PROOF. Use $a=(p-1) /(p+1)$.

REMARKS. Note that a support point of $\overline{S_{H}(U, \Omega)}$ is in $S_{H}(U, \Omega)$ if and only if $\eta=1$ and $\eta=-1$ are both in the support of its probability measure $\mu$.

In general, if $\eta=1$ is not in the support of the measure $\mu \in \mathcal{P}$, then $f$ defined in (2.2) has $\operatorname{Re} f$ bounded above and so $f \in \overline{S_{H}(U, \Omega)} \backslash S_{H}(U, \Omega)$. Indeed, if the support of $\mu$ is contained in $\left\{e^{i \theta}: 0<\beta \leq|\theta| \leq \pi\right\}$, then by (2.1), (2.2), and Theorem 2.8 one has

$$
\begin{aligned}
\operatorname{Re} f(z) & \leq \sup _{\beta / 2 \leq|\alpha| \leq \pi / 2} u_{\alpha}(z)=\sup _{\beta / 2 \leq \alpha \leq \pi / 2} \frac{1}{2}\left[\left(\frac{\pi}{2}-\alpha\right) \tan \alpha+(\pi-\alpha) \cot \alpha\right] \\
& =\frac{(\pi-\beta)}{4} \tan \frac{\beta}{2}+\frac{(2 \pi-\beta)}{4} \cos \frac{\beta}{2}<\infty .
\end{aligned}
$$


Similarly, $\operatorname{Re} f$ is bounded below if $\eta=-1$ is not in the support of $\mu$.

Unfortunately, the support of the measure $\mu$ by itself is not sufficient to tell whether $\operatorname{Re} f$ is bounded. For example, the measure $d \mu(\eta)=\frac{1}{4}|\operatorname{Im} \eta| d \arg \eta$ is supported on the full circle, but its corresponding function

$$
f(z)=\frac{1}{4} \int_{0}^{\pi} \arg \left(\frac{1-e^{-i \theta} z}{1-e^{i \theta} z}\right) d \theta+\frac{i}{2} \arg \frac{1+z}{1-z}
$$

maps $U$ onto the rectangle $\left\{w \in \Omega:|\operatorname{Re} w|<\pi^{2} / 8\right\}$.

Next we shall consider coefficient problems. Let $f=h+\bar{g}$ belong to $\overline{S_{H}(D, \Omega)}$ and have expansions

$$
h(z)=\sum_{k=1}^{\infty} a_{k} z^{k} \quad \text { and } \quad g(z)=\sum_{k=2}^{\infty} b_{k} z^{k}
$$

in a neighborhood of $z=0$. By Theorem 2.3 the leading coefficient $a_{1}$ depends only on $D$ and $a_{1}=\psi_{D}^{\prime}(0)$. Since

$$
h^{\prime}-g^{\prime}=\psi_{D}^{\prime} /\left(1-\psi_{D}^{2}\right),
$$

also the differences $a_{n}-b_{n}$ are independent of $f$ and

$$
a_{n}=b_{n}+\frac{1}{n}\left[\psi_{D}^{\prime} /\left(1-\psi_{D}^{2}\right)\right]_{n-1}, \quad n \geq 2,
$$

where []$_{m}$ denotes the $m$ th coefficient of the function in brackets.

In particular, if $f \in \overline{S_{H}(U, \Omega)}$, then

$$
a_{n}= \begin{cases}b_{n} & \text { if } n \text { is even, } \\ b_{n}+1 / n & \text { if } n \text { is odd }\end{cases}
$$

and if $f \in \overline{S_{H}(\Omega, \Omega)}$, then

$$
a_{n}=b_{n}, \quad n \geq 2 .
$$

THEOREM 2.12. If $f=h+\bar{g}$ belongs to $\overline{S_{H}(U, \Omega)}$ and has expansions (2.5), then $a_{1}=1$ and

$$
\left|a_{n}\right| \leq\left\{\begin{array}{ll}
\frac{1}{2} & \text { if } n \text { is even, } \\
\frac{n+1}{2 n} & \text { if } n \text { is odd, }
\end{array} \quad\left|b_{n}\right| \leq \begin{cases}\frac{1}{2} & \text { if } n \text { is even, } \\
\frac{n-1}{2 n} & \text { if } n \text { is odd, }\end{cases}\right.
$$

for $n \geq 2$. The bounds are sharp.

Proof. If $f=h+\bar{g} \in \bar{S}_{H} \overline{(U, \Omega)}$, then

$$
g^{\prime}(z)=\frac{1}{1-z^{2}} \int_{|\eta|=1} \frac{\eta z}{1-\eta z} d \mu
$$

and so

$$
n b_{n}= \begin{cases}\sum_{k=1}^{n / 2} c_{2 k-1} & \text { if } n \text { is even } \\ \sum_{k=1}^{(n-1) / 2} c_{2 k} & \text { if } n \text { is odd }\end{cases}
$$


where $c_{k}=\int_{|\eta|=1} \eta^{k} d \mu$ are the moments of $\mu$. Since $\left|c_{k}\right| \leq 1$, the bounds for $\left|b_{n}\right|$ follow. Because of (2.6) the bounds for $\left|a_{n}\right|$ follow. Equality occurs in all cases for the functions

$$
f(z)=\operatorname{Re} \frac{z}{1 \mp z}+\frac{i}{2} \arg \frac{1+z}{1-z}
$$

which arise from unit measures at $\eta= \pm 1$.

REMARK. Using (2.8) and (2.6) one can describe the regions of values for coefficients of functions in $\overline{S_{H}(U, \Omega)}$. The region of values of $a_{2 k}$ and $b_{2 k}$ is the convex hull of the curve

$$
\frac{\sin (k t)}{2 k \sin t} e^{i k t}, \quad 0 \leq t \leq 2 \pi,
$$

for $k=1,2,3, \ldots$. Similarly, for $k=1,2,3, \ldots$ the region of values of $a_{2 k+1}$ is the convex hull of the curve

$$
\frac{\sin (k+1) t}{(2 k+1) \sin t} e^{i k t}, \quad 0 \leq t \leq 2 \pi,
$$

and the region of values of $b_{2 k+1}$ is the convex hull of the curve

$$
\frac{\sin (k t)}{(2 k+1) \sin t} e^{i(k+1) t}, \quad 0 \leq t \leq 2 \pi
$$

When $k=1$ these regions are all disks.

For automorphisms of $\Omega$ we have the following coefficient estimates.

THEOREM 2.13. Let $f=h+\bar{g}$ belong to $\overline{S_{H}(\Omega, \Omega)}$ and have expansions (2.5). Then $a_{1}=1$ and

$$
\left|a_{n}\right|=\left|b_{n}\right| \leq \frac{1}{n} C_{n-1} \quad \text { for } n \geq 2
$$

where the constants $C_{n-1}$ are defined by

$$
\frac{1}{1-\tan z}=\sum_{k=0}^{\infty} C_{k} z^{k} \text {. }
$$

The bounds are sharp.

ProOF. We have already observed in Theorem 2.3 that $a_{1}=1$. Since $\psi_{\Omega}(z)=$ $\tanh z$,

$$
g^{\prime}(z)=\int_{|\eta|=1} \frac{\eta \tanh z}{1-\eta \tanh z} d \mu
$$

and so for $n \geq 2$

$$
\begin{aligned}
n\left|b_{n}\right| & =\left|\sum_{k=1}^{n-1}\left[\tanh ^{k} z\right]_{n-1} \int_{|\eta|=1} \eta^{k} d \mu\right| \\
& \leq \sum_{k=1}^{n-1}\left|\left[\tanh ^{k} z\right]_{n-1}\right|=\sum_{k=1}^{n-1}\left[\tan ^{k} z\right]_{n-1} \\
& =\left[\frac{1}{1-\tan z}\right]_{n-1}
\end{aligned}
$$


where we have used the fact that the coefficients of $\tan z$ are real and nonnegative. Because of (2.7) the same bounds are valid for $n a_{n}$. Equality occurs for the functions

$$
f(z)= \pm \frac{1}{2} \arg (1 \pm \sin (2 i z))+i \operatorname{Im} z,
$$

which arise from unit point measures at $\eta=\mp i$.

REMARK. If we use

$$
\frac{x}{2} \cot \frac{x}{2}=1-\sum_{k=1}^{\infty} \frac{B_{k} x^{2 k}}{(2 k) !} \quad \text { and } \quad \sec x=1+\sum_{k=1}^{\infty} \frac{E_{k} x^{2 k}}{(2 k) !}
$$

to generate the Bernoulli numbers $B_{1}=\frac{1}{6}, B_{2}=\frac{1}{30}, B_{3}=\frac{1}{42}, \ldots$ and the Euler numbers $E_{1}=1, E_{2}=5, E_{3}=61, \ldots$, then the identity

$$
\frac{1}{1-\tan z}=\frac{1}{2}+\frac{1}{2} \sec (2 z)+\frac{1}{2} \cot (2 z)-\cot (4 z)
$$

allows us to identify the constants $C_{k}$ in Theorem 2.13. They are

$$
C_{2 k-1}=\frac{\left(64^{k}-16^{k}\right) B_{k}}{(2 k) ! 4} \quad \text { and } \quad C_{2 k}=\frac{2^{2 k-1} E_{k}}{(2 k) !}
$$

for $k=1,2,3, \ldots$. Thus the bounds (2.9) become

$$
\left|a_{2 k}\right|=\left|b_{2 k}\right| \leq \frac{\left(64^{k}-16^{k}\right) B_{k}}{(2 k) ! 8 k} \quad \text { and } \quad\left|a_{2 k+1}\right|=\left|b_{2 k+1}\right| \leq \frac{2^{2 k-1} E_{k}}{(2 k+1) !}
$$

for $k=1,2,3, \ldots$ in terms of the Bernoulli and Euler numbers. In particular,

$$
\begin{array}{ll}
\left|a_{2}\right|=\left|b_{2}\right| \leq \frac{1}{2}, \quad & \left|a_{3}\right|=\left|b_{3}\right| \leq \frac{1}{3}, \\
\left|a_{4}\right|=\left|b_{4}\right| \leq \frac{1}{3}, & \left|a_{5}\right|=\left|b_{5}\right| \leq \frac{1}{3}, \\
\left|a_{6}\right|=\left|b_{6}\right| \leq \frac{16}{45}, & \left|a_{7}\right|=\left|b_{7}\right| \leq \frac{122}{315} .
\end{array}
$$

The next theorem is concerned with estimates of $\left|a_{n}\right|$ and $\left|b_{n}\right|$ that are valid for all domains $D$.

THEOREM 2.14. Let $f=h+\bar{g}$ belong to $\overline{S_{H}(D, \Omega)}$ and have expansions (2.5). Then $a_{1}=\psi_{D}^{\prime}(0)$ and for all $n \geq 2$

$$
\left|a_{n}\right| \leq\left[\frac{(2 n) !}{4(n !)^{2}}+\frac{4^{n}}{8 n}\right] \psi_{D}^{\prime}(0) \quad \text { and } \quad\left|b_{n}\right| \leq\left[\frac{(2 n) !}{4(n !)^{2}}-\frac{4^{n}}{8 n}\right] \psi_{D}^{\prime}(0) \text {. }
$$

The bounds are sharp.

PROOF. It follows from Theorem 2.3 that $a_{1}=\psi_{D}^{\prime}(0)$, and it is no loss of generality to assume $\psi_{D}^{\prime}(0)=1$. Write $f=\tilde{f} \circ \psi_{D}$ where $\tilde{f} \in \overline{S_{H}(U, \Omega)}$ has coefficients $\tilde{a}_{n}$ and $\tilde{b}_{n}$ and $\psi_{D}(z)=z+\sum_{n=2}^{\infty} A_{n} z^{n}$ near $z=0$. Then

$$
a_{n}=\sum_{k=1}^{n} \tilde{a}_{k} \pi_{k n}\left(A_{2}, \ldots, A_{n}\right) \quad \text { and } \quad b_{n}=\sum_{k=2}^{n} \tilde{b}_{k} \pi_{k n}\left(A_{2}, \ldots, A_{n}\right),
$$

where the functions $\pi_{k n}$ are polynomials with nonnegative coefficients.

By a theorem of K. Löwner [7, p. 121] the coefficients $A_{m}$ are dominated by the coefficients of the function

$$
\psi_{D_{0}}(z)=\frac{1-2 z-\sqrt{1-4 z}}{2 z} .
$$


The function $\psi_{D_{0}}$ is inverse to the familiar function $\psi_{D_{0}}^{-1}(z)=z /(1+z)^{2}$ that maps $U$ onto the domain $D_{0}$ consisting of the plane slit along the positive real axis from $1 / 4$ to $\infty$. Since $\psi_{D_{0}}$ has positive coefficients and the estimates of Theorem 2.12 are sharp for the function

$$
f_{0}(z)=\operatorname{Re} \frac{z}{1-z}+\frac{i}{2} \arg \frac{1+z}{1-z},
$$

which has positive coefficients, it follows from (2.10) that $a_{n}$ and $b_{n}$ are dominated by the corresponding coefficients of $f_{0} \circ \psi_{D_{0}}=h_{0}+\bar{g}_{0}$. That is, $a_{n}$ and $b_{n}$ are bounded by the corresponding coefficients of

$$
h_{0}(z)=\frac{1}{4 \sqrt{1-4 z}}-\frac{1}{4}-\frac{1}{8} \log (1-4 z)=\sum_{n=1}^{\infty}\left[\frac{(2 n) !}{4(n !)^{2}}+\frac{4^{n}}{8 n}\right] z^{n}
$$

and

$$
g_{0}(z)=\frac{1}{4 \sqrt{1-4 z}}-\frac{1}{4}+\frac{1}{8} \log (1-4 z)=\sum_{n=1}^{\infty}\left[\frac{(2 n) !}{4(n !)^{2}}-\frac{4^{n}}{8 n}\right] z^{n} .
$$

The bounds are sharp for

$$
f_{0} \circ \psi_{D_{0}}(z)=\frac{1}{2} \operatorname{Re} \frac{1}{\sqrt{1-4 z}}-\frac{1}{2}-\frac{i}{4} \arg (1-4 z) .
$$

Our next application concerns the extremal problems

$$
\max [u(z)-u(-\bar{z})] \text { and } \min [u(z)-u(-\bar{z})]
$$

where $z \in U$ is fixed and $f=u+i v$ varies over $\overline{S_{H}(U, \Omega)}$. Observe that $v(z)=$ $v(-\bar{z})=\frac{1}{2} \arg ((1+z) /(1-z))$ for all $f$.

If $z$ is purely imaginary, then $z=-\bar{z}$ and the extremal problems (2.11) are trivial. Assume therefore that $\operatorname{Re} z \neq 0$.

Since the extremal problems (2.11) are linear, it is sufficient to find the extreme values over the set of extreme points of $\overline{S_{H}(U, \Omega)}$, that is,

$$
\max _{\alpha}\left[u_{\alpha}(z)-u_{\alpha}(-\bar{z})\right] \text { and } \min _{\alpha}\left[u_{\alpha}(z)-u_{\alpha}(-\bar{z})\right] \text {. }
$$

After the substitutions $t=\sin 2 \alpha$ and $w=2 i z /\left(1-z^{2}\right)$ these become

$$
\max _{-1 \leq t \leq 1} \frac{1}{t} \arg (1+t w) \text { and } \min _{-1 \leq t \leq 1} \frac{1}{t} \arg (1+t w),
$$

where $w$ is fixed, $\operatorname{Im} w \neq 0$, and $\arg 1=0$.

LEMMA 2.15. For fixed $\theta, 0<|\theta|<\pi$, let $\phi_{\theta}(t)=\frac{1}{t} \arg \left(1+t e^{i \theta}\right)$ with $\arg (1)=$ 0 and $\phi_{\theta}(0)=\sin \theta$. Then $\phi_{\theta}$ has the following properties.

(a) $\phi_{\theta}$ is a $C^{\infty}$ function on $\mathbf{R}$.

(b) $\lim _{t \rightarrow \pm \infty} \phi_{\theta}(t)=0$.

(c) $\phi_{-\theta}=-\phi_{\theta}$.

(d) $\phi_{\pi-\theta}(t)=\phi_{\theta}(-t)$ for $0<\theta<\pi$, and $\phi_{-\pi-\theta}(t)=\phi_{\theta}(-t)$ for $-\pi<\theta<0$.

(e) $\phi_{\theta}(-|t|) \leq \phi_{\theta}(|t|)$ for $-\pi / 2 \leq \theta<0$ or $\pi / 2 \leq \theta<\pi$, and $\phi_{\theta}(-|t|) \geq \phi_{\theta}(|t|)$ for $-\pi<\theta \leq-\pi / 2$ or $0<\theta \leq \pi / 2$.

(f) The equation $\phi_{\theta}^{\prime}(t)=0$ has exactly one solution $t=\tau(\theta)$ for $0<|\theta|<\pi$.

(g) $\tau(\theta)=\tau(-\theta)<0$ for $0<|\theta|<\pi / 2, \tau(\theta)=\tau(-\theta)>0$ for $\pi / 2<|\theta|<\pi$, and $\tau( \pm \pi / 2)=0$. 
(h) If $0<\theta<\pi$, then $\phi_{\theta}$ is positive, increasing for $-\infty<t<\tau(\theta)$, and decreasing for $\tau(\theta)<t<\infty$. If $-\pi<\theta<0$, then $\phi_{\theta}$ is negative, decreasing for $-\infty<t<\tau(\theta)$ and increasing for $\tau(\theta)<t<\infty$.

ProOF. Properties (a), (b), (c), (d), and (e) follow easily from the definition. In preparation for (f) note that

$$
t^{2} \phi_{\theta}^{\prime}(t)=-\operatorname{Im}\left\{\frac{1}{1+t e^{i \theta}}\right\}-\arg \left(1+t e^{i \theta}\right)
$$

and

$$
t^{3} \phi_{\theta}^{\prime \prime}(t)=\operatorname{Im}\left\{\frac{3+4 t e^{i \theta}}{\left(1+t e^{i \theta}\right)^{2}}\right\}+2 \arg \left(1+t e^{i \theta}\right) .
$$

So if $\tau$ is a critical point, then

$$
\tau^{3} \phi_{\theta}^{\prime \prime}(\tau)=\tau^{3} \phi_{\theta}^{\prime \prime}(\tau)+2 \tau^{2} \phi_{\theta}^{\prime}(\tau)=-\operatorname{Im}\left\{\left[\frac{\tau e^{i \theta}}{1+\tau e^{i \theta}}\right]^{2}\right\}
$$

First restrict $\theta \in(0, \pi / 2)$. If $\phi_{\theta}^{\prime}(\tau)=0$ for a $\tau>0$, then $\phi_{\theta}^{\prime \prime}(\tau)<0$ and we would have a strict local maximum at $\tau$. Only one such point would be possible, and we would have $\phi_{\theta}^{\prime}(0) \geq 0$. But $\phi_{\theta}^{\prime}(0)=-\frac{1}{2} \sin 2 \theta<0$, so that a critical point $\tau \geq 0$ is not possible. Suppose next that $\phi_{\theta}^{\prime}(\tau)=0$ for a $\tau \in(-\cos \theta, 0)$. Then $\phi_{\theta}^{\prime \prime}(\tau)>0$ and we would have a strict local minimum. This, too, is impossible since $\phi_{\theta}$ is positive, $\lim _{t \rightarrow \infty} \phi_{\theta}(t)=0$, and $\phi_{\theta}$ has no local maximum to the right. The point $t=-\cos \theta$ is not a critical point since

$$
\phi_{\theta}^{\prime}(-\cos \theta)=-\left(\sec ^{2} \theta\right)\left[\tan \left(\frac{\pi}{2}-\theta\right)-\left(\frac{\pi}{2}-\theta\right)\right]<0 .
$$

Finally, if $\phi_{\theta}^{\prime}(\tau)=0$ for a $\tau<-\cos \theta$, then $\phi_{\theta}^{\prime \prime}(\tau)<0$ and we have a strict local maximum. Only one such critical point $\tau=\tau(\theta)$ can appear, and so (f) is proved for $\theta \in(0, \pi / 2)$.

If $\theta=\pi / 2$, then $\phi_{\pi / 2}^{\prime \prime}(\tau)=-2 /(1+\tau)^{2}<0$ at each critical point. Hence there is only one. In fact, $\phi_{\pi / 2}^{\prime}(\tau)=0$ if and only if $\tau=0$.

The remaining cases of (f) and properties (g) and (h) now follow by applying properties (c) and (d).

On the basis of Lemma 2.15 we may conclude the following. For $0<\theta<\pi / 2$,

$$
\min _{|t| \leq|w|} \phi_{\theta}(t)=\phi_{\theta}(|w|) \text { and } \max _{|t| \leq|w|} \phi_{\theta}(t)= \begin{cases}\phi_{\theta}(-|w|) & \text { if } \tau(\theta) \leq-|w| \\ \phi_{\theta}(\tau(\theta)) & \text { if } \tau(\theta)>-|w|\end{cases}
$$

For $\pi / 2<\theta<\pi$,

$$
\min _{|t| \leq|w|} \phi_{\theta}(t)=\phi_{\theta}(-|w|) \quad \text { and } \max _{|t| \leq|w|} \phi_{\theta}(t)= \begin{cases}\phi_{\theta}(|w|) & \text { if } \tau(\theta) \geq|w| \\ \phi_{\theta}(\tau(\theta)) & \text { if } \tau(\theta)<|w| .\end{cases}
$$

For $\theta=\pi / 2$,

$$
\min _{|t| \leq|w|} \phi_{\pi / 2}(t)=\phi_{\pi / 2}( \pm|w|) \quad \text { and } \max _{|t| \leq|w|} \phi_{\pi / 2}(t)=\phi_{\pi / 2}(0)=1 .
$$


THEOREM 2.16. Let $f=u+i v$ belong to $\overline{S_{H}(U, \Omega)}$ and set $w=|w| e^{i \theta}=$ $2 i z /\left(1-z^{2}\right)$. For $0<|\theta|<\pi$ define

$$
\begin{aligned}
& A(z)=-\arg \frac{(1-i z)^{2}}{1-z^{2}}, \\
& B(z)= \begin{cases}\arg \frac{(1+i z)^{2}}{1-z^{2}} & \text { if } \tau(\theta) \geq \frac{2|z|}{\left|1-z^{2}\right|}, \\
\frac{2|z|}{\left|1-z^{2}\right|} \phi_{\theta}(\tau(\theta)) & \text { if } \tau(\theta)<\frac{2|z|}{\left|1-z^{2}\right|},\end{cases}
\end{aligned}
$$

where $\phi_{\theta}(t)=(1 / t) \arg \left(1+t e^{i \theta}\right), \phi_{\theta}(0)=\sin \theta$, and $t=\tau(\theta)$ is the unique solution of the equation $\phi_{\theta}^{\prime}(t)=0$. Then

$$
\begin{aligned}
2 \arg (1+i x) & \leq|u(x)-u(-x)| \leq 2 x /\left(1-x^{2}\right) \quad \text { if }-1<x<1, \\
A(z) & \leq|u(z)-u(-\bar{z})| \leq B(z) \quad \text { if } \operatorname{Im} z>0, \quad \text { and } \\
A(\bar{z}) & \leq|u(z)-u(-\bar{z})| \leq B(\bar{z}) \quad \text { if } \operatorname{Im} z<0 .
\end{aligned}
$$

PROOF. If $\operatorname{Re} z=0$, the estimates are all trivial. Assume, therefore, that $\operatorname{Re} z \neq 0$. We shall use the relations

$$
\begin{aligned}
& \min _{-1 \leq t \leq 1} \frac{1}{t} \arg (1+t w)=\min _{|t| \leq|w|}|w| \phi_{\theta}(t) \text { and } \max _{-1 \leq t \leq 1} \frac{1}{t} \arg (1+t w)=\max _{|t| \leq|w|}|w| \phi_{\theta}(t), \\
& A(z)=|w| \phi_{\theta}(-|w|) \text { and } A(\bar{z})=|w| \phi_{\theta}(|w|), \\
& B(z)=\left\{\begin{array}{ll}
|w| \phi_{\theta}(|w|) & \text { if } \tau(\theta) \geq|w|, \\
|w| \phi_{\theta}(\tau(\theta)) & \text { if } \tau(\theta)<|w|,
\end{array} \quad B(\bar{z})= \begin{cases}|w| \phi_{\theta}(-|w|) & \text { if } \tau(\theta) \leq-|w|, \\
|w| \phi_{\theta}(\tau(\theta)) & \text { if } \tau(\theta)>-|w|,\end{cases} \right. \\
& B(-\bar{z})=-B(z) \text { and } B(-z)=-B(\bar{z}) \text {, }
\end{aligned}
$$

and the extrema developed above.

First, if $z=x \in(0,1)$, then $w=\left(2 x /\left(1-x^{2}\right)\right) e^{i \pi / 2}$ and so

$$
2 \arg (1+i x)=|w| \phi_{\pi / 2}( \pm|w|) \leq u(x)-u(-x) \leq|w| \phi_{\pi / 2}(0)=2 x /\left(1-x^{2}\right) .
$$

Furthermore, if $z=x \in(-1,0)$, it follows that $-x \in(0,1)$ and

$$
2 \arg (1-i x) \leq u(-x)-u(x) \leq-2 x /\left(1-x^{2}\right) .
$$

These inequalities can be written more concisely as (a).

Consider next the case $z=|z| e^{i \gamma}$ with $0<\gamma<\pi / 2$. Then $w=2 i z /\left(1-z^{2}\right)=$ $|w| e^{i \theta}$ where $\pi / 2<\theta<\pi$ and we have the bounds

$$
A(z) \leq u(z)-u(-\bar{z}) \leq B(z) .
$$

Furthermore, if $z=|z| e^{i \gamma}$ where $\pi / 2<\gamma<\pi$, then we can apply these estimates to $-\bar{z}$ :

$$
A(-\bar{z}) \leq u(-\bar{z})-u(z) \leq B(-\bar{z}) .
$$

Since $A(-\bar{z})=-A(z)$ and $B(-\bar{z})=-B(z)$, these bounds can be written compactly as (b).

Finally, consider the case $z=|z| e^{i \gamma}$ with $-\pi / 2<\gamma<0$. Then $w=2 i z /\left(1-z^{2}\right)=$ $|w| e^{i \theta}$ where $0<\theta<\pi / 2$ and we have the bounds

$$
A(\bar{z}) \leq u(z)-u(-\bar{z}) \leq B(\bar{z}) .
$$


Furthermore, if $z=|z| e^{i \gamma}$ where $-\pi<\gamma<-\pi / 2$, then we can apply these estimates to $-\bar{z}$ :

$$
A(-z) \leq u(-\bar{z})-u(z) \leq B(-z) .
$$

Since $A(-z)=-A(\bar{z})$ and $B(-z)=-B(\bar{z})$, these bounds can be written as (c).

In each case we remark that the inequalities are sharp.

3. Harmonic mappings of $\widetilde{U}$. First we find the form of harmonic mappings of $\widetilde{U}=\{z:|z|>1\}$ for which $f(\infty)=\lim _{z \rightarrow \infty} f(z)$ exists as $\infty$.

LEMMA 3.1. Let $f$ be a complex-valued, harmonic, orientation-preserving, univalent mapping of $\widetilde{U}$ with $f(\infty)=\infty$. Then $f$ has the representation

$$
f(z)=\alpha z+\beta \bar{z}+A \log |z|+\sum_{k=0}^{\infty} a_{k} z^{-k}+\overline{\sum_{k=1}^{\infty} b_{k} z^{-k}}
$$

where $0 \leq|\beta|<|\alpha|$. In addition, $a=\overline{f_{\bar{z}}} / f_{z}$ is analytic and satisfies $|a(z)|<1$.

PROOF. Since $f$ is harmonic and $f(\infty)=\infty$, it has the representation

$$
f(z)=h(z)+\overline{g(z)}+A \log |z|
$$

where

$$
h(z)=\sum_{k=-\infty}^{\infty} a_{k} z^{-k} \text { and } g(z)=\sum_{k=-\infty}^{\infty} b_{k} z^{-k}
$$

are analytic in $\widetilde{U}$ and $A \in \mathbf{C}$.

The orientation-preserving property implies that the Jacobian $\left|f_{z}\right|^{2}-\left|f_{\bar{z}}\right|^{2}$ is nonnegative, and so

$$
\left|f_{\bar{z}}(z)\right|=\left|g^{\prime}(z)+\bar{A} /(2 z)\right| \leq\left|f_{z}(z)\right|=\left|h^{\prime}(z)+A /(2 z)\right| .
$$

If the latter were to vanish identically, then $f$ would be constant and not univalent. Therefore

$$
a(z)=\frac{2 z g^{\prime}(z)+\bar{A}}{2 z h^{\prime}(z)+A}
$$

is analytic in $\widetilde{U}$ and $|a(z)| \leq 1$. If $|a(z)|=1$ at some point, then the maximum principle would imply $g^{\prime}(z)-e^{2 i \gamma} h^{\prime}(z)=\frac{1}{2}\left(e^{2 i \gamma} A-\bar{A}\right) / z$. On the left the coefficient of $1 / z$ is zero. Therefore $g=e^{2 i \gamma} h+c$, where $c$ is a constant. As a result,

$$
e^{i \gamma}[f(z)-\bar{c}]=2 \operatorname{Re}\left\{e^{i \gamma} h(z)\right\}+A e^{i \gamma} \log |z|
$$

would not cover a full neighborhood of $\infty$. Therefore $|a(z)|<1$ and $|a(\infty)|<1$ for the analytic extension.

The mapping $f$ is a quasiconformal mapping on $|z|>R$ for each $R>1$. By the distortion theorem for such mappings (see [6, Chapter II, §3]) we have $|f(z)|=$ $O\left(|z|^{K}\right)$ as $z \rightarrow \infty$ for each real number $K>(1+|a(\infty)|) /(1-|a(\infty)|)$. It follows that the Fourier coefficients $a_{-n}$ and $b_{-n}$ are all zero for $n>K$. Therefore the singular part of $f$ at $\infty$ is of the form

$$
\sum_{k=-N}^{-1} a_{k} z^{-k}+\overline{\sum_{k=-N}^{-1} b_{k} z^{-k}}+A \log |z|
$$


If $a_{-N} \neq 0$, then $\left|b_{-N}\right|<\left|a_{-N}\right|$ since $|a(\infty)|<1$ and

$$
f\left(R e^{i \theta}\right)=a_{-N} R^{N} e^{i N \theta}\left[1+\left(b_{-N} / a_{-N}\right) e^{-2 i N \theta}+o(1)\right] \quad \text { as } R \rightarrow \infty .
$$

In this case $f$ is univalent only if $N=1$ and we have the conclusion of the lemma. The alternative would be that $f$ has the form

$$
f\left(R e^{i \theta}\right)=A \log R+O(1) \text { as } R \rightarrow \infty,
$$

in which case $f$ could not cover a neighborhood of $\infty$.

By applying an affine post-mapping to $f$ we may normalize $f$ so that $\alpha=1$, $\beta=0$, and $a_{0}=0$. Therefore let $\Sigma_{H}^{\prime}$ be the set of all harmonic, orientationpreserving, univalent mappings

$$
f(z)=h(z)+\overline{g(z)}+A \log |z|
$$

of $\widetilde{U}$, where

$$
h(z)=z+\sum_{k=1}^{\infty} a_{k} z^{-k} \quad \text { and } \quad g(z)=\sum_{k=1}^{\infty} b_{k} z^{-k}
$$

are analytic in $\widetilde{U}$ and $A \in \mathbf{C}$. The subclass with no logarithmic singularity will be denoted by $\Sigma_{H}^{\prime \prime}$ :

$$
\Sigma_{H}^{\prime \prime}=\left\{f \in \Sigma_{H}^{\prime}: A=0\right\} .
$$

The following estimates are essentially a consequence of Schwarz's lemma.

THEOREM 3.2. (a) If $f \in \Sigma_{H}^{\prime}$, then $|A| \leq 2$ and $\left|b_{1}\right| \leq 1$.

(b) If $f \in \Sigma_{H}^{\prime \prime}$, then $\left|b_{1}\right| \leq 1$ and $\left|b_{2}\right| \leq \frac{1}{2}\left(1-\left|b_{1}\right|^{2}\right) \leq \frac{1}{2}$.

PRoOF. We shall use the bounds $\left|\omega_{0}\right| \leq 1$ and $\left|\omega_{1}\right| \leq 1-\left|\omega_{0}\right|^{2}$ for analytic functions $\omega(z)=\omega_{0}+\omega_{1} z^{-1}+\cdots$ in $\widetilde{U}$ that are bounded by one.

If $f \in \Sigma_{H}^{\prime}$ has expansion (3.1), then

$$
\begin{aligned}
a(z)= & \frac{2 z g^{\prime}(z)+\bar{A}}{2 z h^{\prime}(z)+A}=\frac{1}{2} \bar{A} z^{-1}-\left(b_{1}+\frac{1}{4}|A|^{2}\right) z^{-2} \\
& -\left[2 b_{2}-\frac{1}{2} \bar{A} a_{1}-\frac{1}{2} A b_{1}-\frac{1}{8} A|A|^{2}\right] z^{-3}+\cdots
\end{aligned}
$$

is analytic in $\widetilde{U}$ and $|a(z)|<1$ by Lemma 3.1. The maximum principle implies that $\omega(z)=z a(z)$ is also bounded by one, and so $\left|\frac{1}{2} \bar{A}\right| \leq 1$ and $\left.\left.\left|b_{1}+\frac{1}{4}\right| A\right|^{2}|\leq 1-| \frac{1}{2} \bar{A}\right|^{2}$. The latter implies $\left|b_{1}\right| \leq 1$.

If $f$ belongs to $\Sigma_{H}^{\prime \prime}$, then $A=0$,

$$
a(z)=-b_{1} z^{-2}-2 b_{2} z^{-3}+\cdots,
$$

and $\omega(z)=z^{2} a(z)$ is bounded by one. Therefore $\left|b_{1}\right| \leq 1$ and $\left|2 b_{2}\right| \leq 1-\left|b_{1}\right|^{2}$.

The coefficient bounds in Theorem 3.2 are all sharp. Equality in (a) is attained, for example, by the function

$$
f(z)=z-1 / \bar{z}+2 \log |z|,
$$

which maps $\tilde{U}$ onto $\mathbf{C} \backslash\{0\}$. For a proof that $f$ belongs to $\Sigma_{H}^{\prime}$ see Theorem 3.7. In the proof of part (a) we could have observed also that $b_{1}$ must lie in a disk of radius $1-\frac{1}{4}|A|^{2}$ about the point $-\frac{1}{4}|A|^{2}$. 
In (b) the bound for $b_{1}$ is sharp for the function

$$
f(z)=z+e^{i \gamma} / \bar{z}, \quad-\pi \leq \gamma \leq \pi,
$$

which maps $\tilde{U}$ onto the exterior of the (possibly degenerate) circle $|w|=2 \cos (\gamma / 2)$.

In addition, the bound for $b_{2}$ is attained by the function

$$
f(z)=z+1 /\left(2 \bar{z}^{2}\right) \text {. }
$$

REMARK. In contrast to the classical analytic families $S$ and $\Sigma$, the functions above show that support points of $\Sigma_{H}^{\prime}$ and $\Sigma_{H}^{\prime \prime}$ need not be slit mappings. Furthermore, $f(\widetilde{U})$ may not be conformally equivalent to $\widetilde{U}$.

The following lemma contains a distortion estimate for a class of locally quasiconformal mappings.

LEMMA 3.3. Let $f$ be a diffeomorphism of $U$ satisfying

$$
\left|f_{\bar{z}}(z)\right| \leq|z|\left|f_{z}(z)\right| \quad \text { for all } z \in U
$$

$$
f(z)=z+O\left(|z|^{\beta}\right) \quad \text { for some } \beta>1 \text { as } z \rightarrow 0 .
$$

Then for all $z \in U$ we have

$$
|f(z)| \geq|z| /\left[4(1+|z|)^{2}\right] .
$$

In particular, the disk $\left\{w:|w|<\frac{1}{16}\right\}$ is contained in $f(U)$.

ProOF. In their paper [2, Theorem 4.4] Clunie and Sheil-Small proved Lemma 3.3 for harmonic mappings $f=h+\bar{g}$ satisfying (a) and (b). But their proof applies equally well to the present situation. One only replaces $h^{\prime}$ by $f_{z}$ and $g^{\prime}$ by $\overline{f_{\bar{z}}}$.

An immediate consequence is the following distortion theorem for the nonvanishing class

$$
\Sigma_{H}^{0}=\left\{f-c: f \in \Sigma_{H}^{\prime} \text { and } c \notin f(\widetilde{U})\right\} .
$$

THEOREM 3.4. If $f(z)=z-c+A \log |z|+\sum_{k=1}^{\infty} a_{k} z^{-k}+\overline{\sum_{k=1}^{\infty} b_{k} z^{-k}}$ belongs to $\Sigma_{H}^{0}$, then

$$
|f(z)| \leq 4(1+|z|)^{2} /|z| \text { for all } z \in \widetilde{U},
$$

$f(\widetilde{U})$ contains the set $\{w:|w|>16\}$, and $|c| \leq 16$.

ProOF. If $f$ belongs to $\Sigma_{H}^{0}$, then $\tilde{f}(z)=1 / f(1 / z)$ is a diffeomorphism of $U$ that satisfies

$$
\begin{gathered}
\left|\tilde{f}_{\bar{z}}(z)\right| /\left|\tilde{f}_{z}(z)\right|=|a(1 / z)| \leq|z| \quad \text { for } z \in U, \\
\tilde{f}(z)=z-A z^{2} \log z+O\left(|z|^{2}\right) \quad \text { as } z \rightarrow 0 .
\end{gathered}
$$

Therefore Lemma 3.3 applies to $\tilde{f}$, and the first two conclusions follow. In addition,

$$
|-c+A \log R|=\left|\frac{1}{2 \pi} \int_{0}^{2 \pi} f\left(R e^{i \theta}\right) d \theta\right| \leq 4(1+R)^{2} / R
$$

for all $R>1$. Let $R$ approach 1 to obtain $|c| \leq 16$.

The bound for $c$ is equivalent to the following. 
COROLlaRY 3.5. If $f$ belongs to $\Sigma_{H}^{\prime}$, then $f(\widetilde{U})$ contains the set $\{w:|w|>16\}$.

The next result concerns compactness of our families.

THEOREM 3.6. The families $\Sigma_{H}^{0}, \Sigma_{H}^{\prime}$, and $\Sigma_{H}^{\prime \prime}$ are compact with respect to the topology of locally uniform convergence.

PROOF. By Theorem 3.4 the families are locally uniformly bounded families of harmonic functions, hence normal. If $\left\{f_{n}\right\}$ is a convergent sequence in one of these families and $f_{n} \rightarrow f$, then $f$ is harmonic and $f_{z}(\infty)=1$. To see that $f$ is univalent, we use the fact that each $f_{n}$ satisfies $\left(f_{n}\right)_{\bar{z}}=\overline{a_{n}\left(f_{n}\right)_{z}}$ where $a_{n}$ is analytic and satisfies $\left|a_{n}(z)\right| \leq 1 /|z|$. That is, each $f_{n}$ is a $K_{R}$-quasiconformal mapping in $|z|>R, R>1$, with $K_{R}=(R+1) /(R-1)$. Limits of such mappings are either univalent or constant. Since $f_{z}(\infty)=1$, the function $f$ is univalent. Furthermore, the normalizations are preserved.

In the examples following Theorem 3.2 we have seen that it is possible for the diameter of the omitted set $\mathbf{C} \backslash f(\widetilde{U})$ to be zero for $f$ in $\Sigma_{H}^{\prime}$ or $\Sigma_{H}^{\prime \prime}$. The following theorem contains a sharp lower bound for this diameter depending on the coefficient $b_{1}$.

THEOREM 3.7. If $f \in \Sigma_{H}^{\prime}$, then the diameter $D_{f}$ of $\mathbf{C} \backslash f(U)$ satisfies

$$
D_{f} \geq 2\left|1+b_{1}\right| \text {. }
$$

This estimate is sharp for

$$
f(z)=z+b_{1} / \bar{z}+A \log |z|
$$

whenever $\left|b_{1}\right|<1$ and $|A| \leq\left(1-\left|b_{1}\right|^{2}\right) /\left|1+b_{1}\right|,\left|b_{1}\right|=1$ and $A=0$, or $b_{1}=-1$ and $|A| \leq 2$.

ProOF. Let $D_{f}(R)$ be the diameter of $f(|z|=R), R>1$, and let $D_{f}^{*}(R)=$ $\max _{|z|=R}|f(z)-f(-z)|$. Then $D_{f}(R) \searrow D_{f}$ as $R \rightarrow 1$ and $D_{f}(R) \geq D_{f}^{*}(R)$. Since

$$
\begin{aligned}
D_{f}^{*}(R)^{2} & \geq \frac{1}{2 \pi} \int_{0}^{2 \pi}\left|f\left(R e^{i \theta}\right)-f\left(-R e^{i \theta}\right)\right|^{2} d \theta \\
& =4\left[\left|R+b_{1} R^{-1}\right|^{2}+\left|a_{1}\right|^{2} R^{-2}+\sum_{k=1}^{\infty}\left(\left|a_{2 k+1}\right|^{2}+\left|b_{2 k+1}\right|^{2}\right) R^{-2(2 k+1)}\right] \\
& \geq 4\left|R+b_{1} R^{-1}\right|^{2},
\end{aligned}
$$

we conclude that $D_{f} \geq 2\left|1+b_{1}\right|$.

For the given parameters $b_{1}$ and $A$ the function (3.2) maps $\widetilde{U}$ onto the exterior of the circle $|w|=\left|1+b_{1}\right|$ and shows that the bound is sharp. To see that the mapping is univalent either observe that it maps the circles $|z|=R, R>1$, onto an increasing family of (possibly nonconcentric) circles or note that it is a local homeomorphism since its Jacobian is positive and is one-to-one on circles near $\partial \tilde{U}$.

Note that we have proved the apparently stronger bound

$$
D_{f} \geq 2 \sqrt{\left|1+b_{1}\right|^{2}+\sum_{k=1}^{\infty}\left(\left|a_{2 k-1}\right|^{2}+\left|b_{2 k+1}\right|^{2}\right)} .
$$

Corresponding to the classical area theorem is the following. 
THEOREM 3.8. If $f \in \Sigma_{H}^{\prime}$ has expansion (3.1), then

$$
\sum_{k=1}^{\infty} k\left(\left|a_{k}\right|^{2}-\left|b_{k}\right|^{2}\right) \leq 1+2 \operatorname{Re} b_{1} .
$$

Equality occurs if and only if $\mathbf{C} \backslash f(\widetilde{U})$ has area zero.

PROOF. The area of the omitted set is

$$
\begin{aligned}
\lim _{R \rightarrow 1} \frac{1}{2 i} \int_{|z|=R} \bar{f} d f & =\lim _{R \rightarrow 1}\left[\frac{1}{2 i} \int_{|z|=R} \bar{h} h^{\prime} d z+\frac{1}{2 i} \int_{|z|=R} g \overline{g^{\prime}} d \bar{z}+2 \pi \operatorname{Re} b_{1}\right] \\
& =\pi\left[1-\sum_{k=1}^{\infty} k\left(\left|a_{k}\right|^{2}-\left|b_{k}\right|^{2}\right)+2 \operatorname{Re} b_{1}\right] \geq 0 .
\end{aligned}
$$

REMARKS. (1) Since the Jacobian $\left|f_{z}\right|^{2}-\left|f_{\bar{z}}\right|^{2}$ is nonnegative, we have also

$$
1+\sum_{k=1}^{\infty} k^{2}\left(\left|a_{k}\right|^{2}-\left|b_{k}\right|^{2}\right) \geq 0 .
$$

(2) In the next section we shall see that functions $f \in \Sigma_{H}^{\prime}$ which map $\widetilde{U}$ onto complements of real line segments satisfy

$$
a_{1}=1+b_{1} \text { and } a_{k}=b_{k} \text { for } k \geq 2 .
$$

4. Mappings onto complements of real line segments. We now restrict our attention to functions in $\Sigma_{H}^{\prime}$ or $\Sigma_{H}^{\prime \prime}$ that map $\widetilde{U}$ onto complements of (possibly degenerate) real line segments. Denote

$$
\Sigma_{H \mathbf{R}}^{\prime}=\left\{f \in \Sigma_{H}^{\prime}: \mathbf{C} \backslash f(\widetilde{U}) \subset \mathbf{R}\right\} \quad \text { and } \quad \Sigma_{H \mathbf{R}}^{\prime \prime}=\left\{f \in \Sigma_{H}^{\prime \prime}: \mathbf{C} \backslash f(\widetilde{U}) \subset \mathbf{R}\right\}
$$

where $\mathbf{R}$ is the real line in the complex plane. Of course, $\Sigma_{H \mathbf{R}}^{\prime \prime} \subset \Sigma_{H \mathbf{R}}^{\prime}$.

For $f=u+i v$ in $\Sigma_{H \mathbf{R}}^{\prime}$ we have necessarily $v=0$ on $\partial \widetilde{U}$, and the normalization at $\infty$ gives

$$
v(z)=\operatorname{Im} z+r(z)+(\operatorname{Im} A) \log |z|
$$

where $r$ is harmonic and vanishes at infinity. Solving the Dirichlet problem for $r$, we conclude that $r(z)=\operatorname{Im}\{1 / z\}$ and therefore

$$
v(z)=\operatorname{Im}\{z+1 / z\}+(\operatorname{Im} A) \log |z| .
$$

In the representation (3.1) it follows that $\operatorname{Im}\{h(z)+\overline{g(z)}\}=\operatorname{Im}\{z+1 / z\}$. Taking derivatives with respect to $z$, we obtain

$$
h^{\prime}(z)-g^{\prime}(z)=1-z^{-2} .
$$

In Lemma 3.1 we proved that

$$
a(z)=\frac{2 z g^{\prime}(z)+\bar{A}}{2 z h^{\prime}(z)+A}
$$

is analytic in $\widetilde{U}$ and satisfies $|a(z)|<1$. Therefore $p=(1+a) /(1-a)$ is analytic and has positive real part. Since $p(z)=1+\bar{A} / z+\cdots$, we have the Herglotz representation

$$
p(z)=\int_{|\eta|=1} \frac{1+\eta / z}{1-\eta / z} d \mu
$$


for some probability measure $\mu$ on $|\eta|=1$; in addition, $\bar{A}=2 \int_{|\eta|=1} \eta d \mu$.

We will use the identities

$$
\begin{aligned}
h^{\prime}(z)+g^{\prime}(z) & =\frac{1+a(z)}{1-a(z)}\left[h^{\prime}(z)-g^{\prime}(z)+\frac{i(\operatorname{Im} A)}{z}\right]-\frac{\operatorname{Re} A}{z} \\
& =p(z)\left[1-\frac{1}{z^{2}}+\frac{i(\operatorname{Im} A)}{z}\right]-\frac{\operatorname{Re} A}{z} \\
& =\int_{|\eta|=1}\left(\frac{1+\eta / z}{1-\eta / z}\left[1-\frac{1}{z^{2}}+\frac{i(\operatorname{Im} A)}{z}\right]-\frac{\operatorname{Re} A}{z}\right) d \mu \\
& =\int_{|\eta|=1}\left(1+\frac{2 \eta-\bar{A}}{z}+\frac{\left(2 \eta^{2}-1+2 \eta i \operatorname{Im} A\right) z-\eta}{z^{2}(z-\eta)}\right) d \mu \\
& =1+\int_{|\eta|=1} \frac{\left(2 \eta^{2}-1+2 \eta i \operatorname{Im} A\right) z-\eta}{z^{2}(z-\eta)} d \mu
\end{aligned}
$$

to represent

$$
\begin{aligned}
u(z)-(\operatorname{Re} A) \log |z| & =\operatorname{Re}\{h(z)+g(z)\} \\
= & \operatorname{Re}\left\{z+\int_{\infty}^{z}\left[h^{\prime}(z)-1+g^{\prime}(z)\right] d z\right\} \\
= & \operatorname{Re}\left\{z+\int_{|\eta|=1} \int_{\infty}^{z} \frac{\left(2 \eta^{2}-1+2 \eta i \operatorname{Im} A\right) z-\eta}{z^{2}(z-\eta)} d z d \mu\right\} \\
= & \operatorname{Re}\left\{z+\int_{|\eta|=1}\left[2\left(\eta-\frac{1}{\eta}+i \operatorname{Im} A\right) \log \left(1-\frac{\eta}{z}\right)-\frac{1}{z}\right] d \mu\right\} \\
= & \operatorname{Re}\left\{z-\frac{1}{z}\right\}-\int_{|\eta|=1} 2 \operatorname{Im}\{2 \eta+A\} \arg \left(1-\frac{\eta}{z}\right) d \mu .
\end{aligned}
$$

Therefore

$$
f(z)=u(z)+i v(z)=z-\frac{1}{\bar{z}}+A \log |z|-\int_{|\eta|=1} 2 \operatorname{Im}\{2 \eta+A\} \arg \left(1-\frac{\eta}{z}\right) d \mu
$$

Denote

$$
\begin{array}{r}
\mathcal{F}^{\prime}=\left\{f: f(z)=z-\frac{1}{\bar{z}}+A \log |z|-\int_{|\eta|=1} 2 \operatorname{Im}\{2 \eta+A\} \arg \left(1-\frac{\eta}{z}\right) d \mu,\right. \\
\left.A=2 \int_{|\eta|=1} \bar{\eta} d \mu, \mu \in \mathcal{P}\right\}
\end{array}
$$

and

$$
\mathcal{F}^{\prime \prime}=\left\{f \in \mathcal{F}^{\prime}: A=2 \int_{|\eta|=1} \bar{\eta} d \mu=0\right\} .
$$

What we have derived is that

$$
\Sigma_{H \mathbf{R}}^{\prime} \subset \mathcal{F}^{\prime} \text { and } \Sigma_{H \mathbf{R}}^{\prime \prime} \subset \mathcal{F}^{\prime \prime}
$$

The next theorem shows that we even have equality. 
THEOREM 4.1. We have $\Sigma_{H \mathbf{R}}^{\prime}=\mathcal{F}^{\prime}$ and $\Sigma_{H \mathbf{R}}^{\prime \prime}=\mathcal{F}^{\prime \prime}$.

ProOF. Let $f \in \mathcal{F}^{\prime}$. Then $f$ is harmonic in $\widetilde{U}$ and has the required normalization at infinity. By reversing the steps in the derivation of (4.1) one finds that $a=\overline{f_{\bar{z}}} / f_{z}$ satisfies $|a|<1$ in $\tilde{U}$ and that

$$
f_{z}=\left(1-\frac{1}{z^{2}}+\frac{i \operatorname{Im} A}{z}\right) /(1-a)
$$

The latter is nonzero in $\widetilde{U}$ since $|A| \leq 2$. Therefore $f$ is locally univalent and orientation-preserving.

Write

$$
f(z)=F(z)-\int_{|\eta|=1} 2 \operatorname{Im}\{2 \eta+A\} \arg \left(1-\frac{\eta}{z}\right) d \mu, \quad A=2 \int_{|\eta|=1} \bar{\eta} d \mu,
$$

where $F(z)=z-1 / \bar{z}+A \log |z|$. Since $|A| \leq 2$, the function $F$ belongs to $\Sigma_{H}^{\prime}$ by Theorem 3.7. In particular, $F$ is univalent and maps $\widetilde{U}$ onto $\mathbf{C} \backslash\{0\}$.

Since $\operatorname{Im} f=\operatorname{Im} F$, the function $f \circ F^{-1}$ carries horizontal lines into themselves. That is, $f \circ F^{-1}(\sigma+i \tau)=\phi(\sigma, \tau)+i \tau$. We shall show that $\partial \phi / \partial \sigma>0$. Then it will follow that both $f \circ F^{-1}$ and $f$ are univalent.

Using the Jacobian $J_{F}=\left|F_{z}\right|^{2}-\left|F_{\bar{z}}\right|^{2}$, which is positive, and the identity

$$
\frac{\partial F^{-1}}{\partial \sigma}=\frac{2}{i}\left(\frac{\partial \operatorname{Im} F}{\partial \bar{z}}\right) / J_{F}
$$

we compute

$$
\frac{\partial \phi}{\partial \sigma}=2 \operatorname{Re}\left\{\frac{\partial \operatorname{Re} f}{\partial z} \cdot \frac{\partial F^{-1}}{\partial \sigma}\right\}=\frac{4}{J_{F}} \operatorname{Im}\left\{\frac{\partial \operatorname{Re} f}{\partial z} \cdot \frac{\partial \operatorname{Im} F}{\partial \bar{z}}\right\} .
$$

Aided again by the development of (4.1), one finds that

$$
\frac{\partial \operatorname{Re} f}{\partial z}=\frac{p}{2}\left[1-\frac{1}{z^{2}}+\frac{i \operatorname{Im} A}{z}\right]
$$

for some analytic function $p$ of positive real part, and

$$
\frac{\partial \operatorname{Im} F}{\partial \bar{z}}=\frac{i}{2}\left[1-\frac{1}{\bar{z}^{2}}-\frac{i \operatorname{Im} A}{\bar{z}}\right] .
$$

Therefore

$$
\frac{\partial \phi}{\partial \sigma}=\frac{1}{J_{F}}\left|1-\frac{1}{z^{2}}+\frac{i \operatorname{Im} A}{z}\right|^{2} \operatorname{Re} p>0,
$$

and so $f$ is univalent.

Finally, $\operatorname{Im} f(z)=\left(1-1 /|z|^{2}\right) \operatorname{Im} z+(\operatorname{Im} A) \log |z| \rightarrow 0$ as $|z| \rightarrow 1 ;$ thus $\mathbf{C} \backslash f(\widetilde{U})$ is real and $f \in \Sigma_{H \mathbf{R}}^{\prime}$. The same proof also implies $\mathcal{F}^{\prime \prime} \subset \Sigma_{H \mathbf{R}}^{\prime \prime}$.

The following corollary is a consequence of the corresponding properties of $P$.

COROLLARY 4.2. $\Sigma_{H \mathbf{R}}^{\prime}$ is compact, and $\Sigma_{H \mathbf{R}}^{\prime \prime}$ is a compact convex subset. $f$ is a support point of $\Sigma_{H \mathbf{R}}^{\prime \prime}$ if and only if $f$ is of the form

$$
f(z)=z-\frac{1}{\bar{z}}-\sum_{k=1}^{N} 4 \lambda_{k}\left(\operatorname{Im} \eta_{k}\right) \arg \left(1-\frac{\eta_{k}}{z}\right),
$$

where $\lambda_{k} \geq 0,\left|\eta_{k}\right|=1, \sum_{k=1}^{N} \lambda_{k}=1$, and $\sum_{k=1}^{N} \lambda_{k} \eta_{k}=0$. 
REMARKS. (1) $\Sigma_{H \mathbf{R}}^{\prime}$ is apparently not a convex family since $\operatorname{Im} A=$ $-2 \int_{|\eta|=1} \operatorname{Im} \eta d \mu$ appears also in the integrand. The subset of $\Sigma_{H \mathbf{R}}^{\prime}$ consisting of functions for which $A$ is real is convex. One can easily develop a theory for it that parallels the theory for $\Sigma_{H \mathbf{R}}^{\prime \prime}$.

(2) Let $a=\overline{f_{\bar{z}}} / f_{z}$. The harmonic mappings $f \in \Sigma_{H \mathbf{R}}^{\prime}$ correspond to analytic functions $a$ in $\widetilde{U}$ with $|z a(z)| \leq 1$. Furthermore, mappings $f \in \Sigma_{H \mathbf{R}}^{\prime \prime}$ correspond to analytic functions $a$ in $\widetilde{U}$ with $\left|z^{2} a(z)\right| \leq 1$. Moreover, $f$ is a support point of $\Sigma_{H \mathbf{R}}^{\prime \prime}$ if and only if, in addition, $a$ is a finite Blaschke product.

The function $f(z)=z-1 / \bar{z}$ shows that the diameter $D_{f}$ of $\mathbf{C} \backslash f(\tilde{U})$ satisfies

$$
\min _{\Sigma_{H}^{\prime}} D_{f}=\min _{\Sigma_{H}^{\prime \prime}} D_{f}=\min _{\Sigma_{H \mathbf{R}}^{\prime}} D_{f}=\min _{\Sigma_{H \mathbf{R}}^{\prime \prime}} D_{f}=0 .
$$

We are now concerned with $\max D_{f}$.

THEOREM 4.3. The diameter $D_{f}$ of $\mathbf{C} \backslash f(\tilde{U})$ satisfies

$$
\max _{\Sigma_{H \mathbf{R}}^{\prime}} D_{f}=\max _{\Sigma_{H \mathbf{R}}^{\prime \prime}} D_{f}=2 \pi
$$

Equality occurs if and only if

$$
f(z)=z-\frac{1}{\bar{z}}+2 \arg \left(\frac{1+i / z}{1-i / z}\right) .
$$

Proof. Let $f=u+i v \in \Sigma_{H \mathbf{R}}^{\prime}, z_{1}, z_{2} \in \tilde{U}$, and $\left|\eta_{1}\right|=\left|\eta_{2}\right|=1$. Since $\operatorname{Im}\left\{f\left(z_{k}\right)\right\} \rightarrow 0$ as $z_{k} \rightarrow \eta_{k}$, the diameter is $D_{f}=\sup _{\eta_{1}, \eta_{2}} \Delta\left(\eta_{1}, \eta_{2}\right)$, where

$$
\begin{aligned}
\Delta\left(\eta_{1}, \eta_{2}\right) & =\limsup _{z_{k} \rightarrow \eta_{k}}\left[u\left(z_{1}\right)-u\left(z_{2}\right)\right] \\
& =\limsup _{z_{k} \rightarrow \eta_{k}} \int_{|\eta|=1}-2 \operatorname{Im}\{2 \eta+A\} \arg \left(\frac{1-\eta / z_{1}}{1-\eta / z_{2}}\right) d \mu .
\end{aligned}
$$

The functions $\arg \left(\left(1-\eta / z_{1}\right) /\left(1-\eta / z_{2}\right)\right)$ of $\eta$ are bounded as $z_{k} \rightarrow \eta_{k}$ and so by Fatou's lemma we may estimate $\Delta\left(\eta_{1}, \eta_{2}\right)$ above by interchanging lim sup and $\int$.

Next, the points $\eta_{1}$ and $\eta_{2}$ partition the circle $|\eta|=1$ into disjoint $\operatorname{arcs} I_{1}$ and $I_{2}$ which we index so that $\arg \left(\left(1-\eta / \eta_{1}\right) /\left(1-\eta / \eta_{2}\right)\right)$ has a constant value $\alpha$ for $\eta$ on the interior of $I_{1}$ and $\alpha+\pi$ for $\eta$ on the interior of $I_{2}$. Then

$$
\Delta\left(\eta_{1}, \eta_{2}\right) \leq-2 \alpha \int_{I_{1}} \operatorname{Im}\{2 \eta+A\} d \mu-2(\alpha+\pi) \int_{I_{2}} \operatorname{Im}\{2 \eta+A\} d \mu .
$$

We have tacitly included the points $\eta_{1}$ and $\eta_{2}$ in the appropriate $\operatorname{arcs} I_{1}$ or $I_{2}$ in order to bound the limit superior.

Since $\int_{|\eta|=1} \operatorname{Im}\{2 \eta+A\} d \mu=0$, it follows that

$$
\begin{aligned}
\Delta\left(\eta_{1}, \eta_{2}\right) & \leq-2 \pi \int_{I_{2}} \operatorname{Im}\{2 \eta+A\} d \mu=-4 \pi \int_{I_{2}} \operatorname{Im} \eta d \mu-2 \pi \mu\left(I_{2}\right) \operatorname{Im} A \\
& =4 \pi\left\{\mu\left(I_{2}\right) \int_{I_{1}} \operatorname{Im} \eta d \mu-\left[1-\mu\left(I_{2}\right)\right] \int_{I_{2}} \operatorname{Im} \eta d \mu\right\} \\
& \leq 4 \pi\left\{\mu\left(I_{2}\right) \mu\left(I_{1}\right)+\left[1-\mu\left(I_{2}\right)\right] \mu\left(I_{2}\right)\right\} \\
& =8 \pi x(1-x)
\end{aligned}
$$


where $x=\mu\left(I_{2}\right)$. Since $x(1-x) \leq 1 / 4$, we have $\Delta\left(\eta_{1}, \eta_{2}\right) \leq 2 \pi$. Therefore the diameter satisfies $D_{f} \leq 2 \pi$.

Equality can occur only if $x=\mu\left(I_{2}\right)=1 / 2$ and $1-x=\mu\left(I_{1}\right)=1 / 2$ and also only if the measure is concentrated at $\pm i$. Thus equality occurs only if the measure has mass $1 / 2$ at each of the points $\pm i$. This yields the indicated extremal function, which maps $\widetilde{U}$ onto the complement of the real interval $[-\pi, \pi]$. Since the extremal function belongs even to $\Sigma_{H \mathbf{R}}^{\prime \prime}$, the maximum of $D_{f}$ over $\Sigma_{H \mathbf{R}}^{\prime \prime}$ is also $2 \pi$.

Our next theorem answers the question of how far the endpoints of the omitted slit can move.

THEOREM 4.4. If $f \in \Sigma_{H \mathbf{R}}^{\prime}$ and $f(\widetilde{U})=\mathbf{C} \backslash[a, b]$, then we have

$$
-R_{0} \leq a \leq 0 \leq b \leq R_{0}
$$

where $R_{0}=\max _{0 \leq x \leq \pi} 2 x \sin x=2 x_{0} \sin x_{0} \approx 3.6394$ and $x_{0} \approx 2.0288$. The equality $b=R_{0}$ occurs for the function

$$
f_{0}(z)=z-\frac{1}{\bar{z}}-2\left(\cos x_{0}\right) \log |z|-2\left(\sin x_{0}\right) \arg \left(\frac{1+e^{-i x_{0}} / z}{1+e^{i x_{0}} / z}\right)
$$

and the equality $a=-R_{0}$ occurs for $-f_{0}(-z)$. The equalities $a=0$ and $b=0$ occur, for example, for $f(z)=z-1 / \bar{z}$.

Proof. If $f \in \Sigma_{H \mathbf{R}}^{\prime}$, then the mean values

$$
\frac{1}{2 \pi} \int_{0}^{2 \pi} f\left(R e^{i \theta}\right) d \theta=A \log R \rightarrow 0 \quad \text { as } R \rightarrow 1,
$$

and so it is clear that $a \leq 0 \leq b$. Write

$$
f(z)=F(z)-\int_{|\eta|=1} 2 \operatorname{Im}\{2 \eta+A\} \arg (1-\eta / z) d \mu
$$

where

$$
F(z)=z-1 / \bar{z}+A \log |z| .
$$

The function $F$ belongs to $\Sigma_{H}^{\prime}$ by Theorem 3.7 and $\mathbf{C} \backslash F(\tilde{U})=\{0\}$. If $F(z)$ approaches the origin on the positive real axis, then $z$ approaches a point $e^{i \beta}$ with $\cos \beta \geq 0$; in addition, $\operatorname{Im} A=-2 \sin \beta$. It follows from the proof of Theorem 4.1 that the tip of the slit

$$
b=\lim _{t \rightarrow 0^{+}} f \circ F^{-1}(t)=-4 \lim _{t \rightarrow 0^{+}} \int_{|\eta|=1}(\operatorname{Im} \eta-\sin \beta) \arg \left(1-\eta / F^{-1}(t)\right) d \mu .
$$

On the interval $(\beta, \beta+2 \pi)$ the function $\arg \left(1-e^{i \theta} / e^{i \beta}\right)=\frac{1}{2}(\theta-\beta+m \pi)$ for some odd integer $m$. Therefore

$$
b \leq-2 \int_{\beta}^{\beta+2 \pi}(\sin \theta-\sin \beta)(\theta-\beta+m \pi) d \tilde{\mu}=-2 \int_{\beta}^{\beta+2 \pi}(\sin \theta-\sin \beta) \theta d \tilde{\mu}
$$

for an appropriate probability measure $\tilde{\mu}$ on $[\beta, \beta+2 \pi]$. Thus

$$
b \leq \sup _{-\pi / 2 \leq \beta \leq \pi / 2} \max _{\mu \in \mathcal{P}_{\beta}}-2 \int_{\beta}^{\beta+2 \pi} \theta(\sin \theta-\sin \beta) d \mu
$$


where $P_{\beta}$ is the set of probability measures on $[\beta, \beta+2 \pi]$ with the constraint $\int_{\beta}^{\beta+2 \pi} \sin \theta d \mu=\sin \beta$.

Since finitely discrete probability measures are weakly dense in $P$, we consider the Lagrangian

$$
L=-2 \sum_{k=1}^{N} \lambda_{k} \theta_{k}\left(\sin \theta_{k}-\sin \beta\right)-B\left(\sum_{k=1}^{N} \lambda_{k}-1\right)-C\left(\sum_{k=1}^{N} \lambda_{k} \sin \theta_{k}-\sin \beta\right)
$$

where we assume that $\lambda_{k}>0$. Differentiation with respect to $\lambda_{k}$ gives

$$
\frac{\partial L}{\partial \lambda_{k}}=-2 \theta_{k}\left(\sin \theta_{k}-\sin \beta\right)-B-C \sin \theta_{k}=0
$$

If we denote $M=\max _{\rho_{\beta}}-2 \int_{\beta}^{\beta+2 \pi} \theta(\sin \theta-\sin \beta) d \mu$, then the $\operatorname{sum} \sum_{k=1}^{N} \lambda_{k} \partial L / \partial \lambda_{k}$ $=0$ becomes $M=B+C \sin \beta$. Using this to eliminate $B$ in (4.2), we obtain

$$
\left(C+2 \theta_{k}\right)\left(\sin \theta_{k}-\sin \beta\right)=-M \text {. }
$$

We may restrict our attention to $\beta$ for which $M>0$. In this case we have $C+2 \theta_{k} \neq 0$ and $\sin \theta_{k} \neq \sin \beta$. In particular, the endpoints $\beta$ and $\beta+2 \pi$ are not values for $\theta_{k}$.

Differentiation of $L$ with respect to $\theta_{k}$ gives

$$
\frac{-1}{\lambda_{k}} \frac{\partial L}{\partial \theta_{k}}=\left(C+2 \theta_{k}\right) \cos \theta_{k}+2\left(\sin \theta_{k}-\sin \beta\right)=0 .
$$

Together with (4.3), this implies

$$
\left(\sin \theta_{k}-\sin \beta\right)^{2}=\frac{1}{2} M \cos \theta_{k} .
$$

From this it follows that $\cos \theta_{k}>0$. In addition, since the functions $(x-\sin \beta)^{2}$ and $\frac{1}{2} M \sqrt{1-x^{2}}$ are concave in opposite directions, there are at most two values for $x=\sin \theta_{k}$ that satisfy (4.4). Furthermore, since $\cos \theta_{k}>0$, there are at most two values $\theta_{k}$ in $(\beta, \beta+2 \pi)$ that satisfy (4.4). If there were only one value $\theta_{1}$, then the constraint would force $\sin \theta_{1}=\sin \beta$ and $M=0$. Thus there are exactly two values $\theta_{1}$ and $\theta_{2}$, which we may index so that $\sin \theta_{1}<\sin \beta<\sin \theta_{2}$ and $0<\theta_{1}-\theta_{2}<2 \pi$.

If $\lambda=\lambda_{1}$, then $\sin \beta=\lambda \sin \theta_{1}+(1-\lambda) \sin \theta_{2}$ and (4.3) for $k=1,2$ becomes

$$
\begin{array}{r}
(1-\lambda)\left(C+2 \theta_{1}\right)\left(\sin \theta_{2}-\sin \theta_{1}\right)=M, \\
-\lambda\left(C+2 \theta_{2}\right)\left(\sin \theta_{2}-\sin \theta_{1}\right)=M .
\end{array}
$$

Therefore we have

$$
\begin{aligned}
M & =2 \lambda(1-\lambda)\left(\theta_{1}-\theta_{2}\right)\left(\sin \theta_{2}-\sin \theta_{1}\right) \\
& =-4 \lambda(1-\lambda)\left(\theta_{1}-\theta_{2}\right) \cos \left(\frac{\theta_{1}+\theta_{2}}{2}\right) \sin \left(\frac{\theta_{1}-\theta_{2}}{2}\right) \\
& \leq \max _{\substack{0 \leq \lambda \leq 1 \\
0 \leq x \leq \pi}} 8 \lambda(1-\lambda) x \sin x \\
& =\max _{0 \leq x \leq \pi} 2 x \sin x=2 x_{0} \sin x_{0} .
\end{aligned}
$$

Thus $b \leq R_{0}=2 x_{0} \sin x_{0}$. Equality occurs for the function $f_{0}$ given in the statement of the theorem because $f_{0}(1)=R_{0}$.

The inequality $-R_{0} \leq a$ follows by applying what has been proved to $-f(-z)$.

For the class $\Sigma_{H \mathbf{R}}^{\prime \prime}$ the endpoints of the omitted slit cannot move as far as in Theorem 4.4. 
THEOREM 4.5. If $f \in \Sigma_{H \mathbf{R}}^{\prime \prime}$ and $f(\tilde{U})=\mathbf{C} \backslash[a, b]$, then we have

$$
-\pi \leq a \leq 0 \leq b \leq \pi .
$$

Both equalities $a=-\pi$ and $b=\pi$ occur for the function

$$
f(z)=z-1 / \bar{z}+2 \arg ((1+i / z) /(1-i / z)) .
$$

PROOF. The proof begins as in Theorem 4.4, except that now $\beta=0$ and we have the additional constraint $\int_{0}^{2 \pi} \cos \theta d \mu=0$. Consider therefore the Lagrangian

$$
L=-2 \sum_{k=1}^{N} \lambda_{k} \theta_{k} \sin \theta_{k}-B\left(\sum_{k=1}^{N} \lambda_{k}-1\right)-C \sum_{k=1}^{N} \lambda_{k} \sin \theta_{k}-D \sum_{k=1}^{N} \lambda_{k} \cos \theta_{k},
$$

where all $\lambda_{k}>0$. As before,

$$
\partial L / \partial \lambda_{k}=-2 \theta_{k} \sin \theta_{k}-B-C \sin \theta_{k}-D \cos \theta_{k}=0,
$$

and if $M$ denotes the maximum of our functional, then the sum $\sum_{k=1}^{N} \lambda_{k} \partial L / \partial \lambda_{k}=$ 0 implies $M=B$. Therefore (4.5) becomes

$$
\left(C+2 \theta_{k}\right) \sin \theta_{k}+D \cos \theta_{k}=-M
$$

for each $k$.

If $\theta_{k} \neq 0,2 \pi$, then differentiation of $L$ with respect to $\theta_{k}$ gives

$$
\frac{-1}{\lambda_{k}} \frac{\partial L}{\partial \theta_{k}}=\left(C+2 \theta_{k}\right) \cos \theta_{k}+(2-D) \sin \theta_{k}=0 .
$$

Using (4.6) to eliminate $C$ in (4.7), we obtain the quadratic equation

$$
2 \cos ^{2} \theta_{k}+M \cos \theta_{k}+D-2=0
$$

in $\cos \theta_{k}$ for $\theta_{k} \neq 0,2 \pi$.

If either endpoint 0 or $2 \pi$ were to satisfy (4.6), then $D=-M$ and equation (4.8) would imply that $\cos \theta_{k}$ equals 1 or $-1-M / 2$ for $\theta_{k} \neq 0,2 \pi$. Since we may assume $M>0$, there could be no $\theta_{k} \neq 0,2 \pi$ and so the constraint $\sum_{k=1}^{N} \lambda_{k} \cos \theta_{k}=0$ would be violated. Thus neither endpoint is possible, and (4.7) and (4.8) are valid for all $k$.

It takes more than one $\theta_{k}$ to satisfy the constraints. If there are only two, we may index them so that $\theta_{2}=\theta_{1}+\pi$. Substituting both into (4.7) and adding the two equations, we find that $\cos \theta_{1}=0$ or $\theta_{1}=\pi / 2$ and $\theta_{2}=3 \pi / 2$. In this case the constraints require that $\lambda_{1}=\lambda_{2}=1 / 2$, and we are led to the extremal function and bound indicated in the statement of the theorem.

It remains to eliminate the case of three or more points $\theta_{k}$. In this case the quadratic equation (4.8) requires that two of them have the same cosine, say $\theta_{1}$ and $\theta_{2}=2 \pi-\theta_{1}$. Substituting both of these points into (4.6) and subtracting, or into (4.7) and adding, we find that $C=-2 \pi$. Therefore equation (4.7) becomes

$$
2\left(\theta_{k}-\pi\right) \cot \theta_{k}=D-2
$$

for each $\theta_{k} \neq \pi$. Since the solution $-\frac{1}{4} M-\frac{1}{4} \sqrt{M^{2}-8 D+16}$ of the quadratic equation (4.8) must be at least -1 , we also have $D \geq M$. Thus

$$
2\left(\theta_{k}-\pi\right) \cot \theta_{k} \geq M-2 \geq \pi-2>0
$$


or $\theta_{k}=\pi$. As a result, $\cos \theta_{k}$ is negative for all $k$, and so the constraint

$$
\sum_{k=1}^{N} \lambda_{k} \cos \theta_{k}=0
$$

is not satisfied.

If $f=u+i v$ belongs to $\Sigma_{H \mathbf{R}}^{\prime}$, then $v(z)-v(-\bar{z})=0$. Therefore we may obtain a measure of distortion for $f$ by considering $u(z)-u(-z)$.

(a)

THEOREM 4.6. If $z \in \widetilde{U}$ and $\operatorname{Re} z>0$, then

$\max _{\Sigma_{H \mathbf{R}}^{\prime}}[u(z)-u(-\bar{z})]=\max _{\Sigma_{H \mathbf{R}}^{\prime \prime}}[u(z)-u(-\bar{z})]=2(\operatorname{Re} z)\left(1-\frac{1}{|z|^{2}}\right)+4 \arg \left(\frac{1+i / z}{1-i / z}\right)$,

$$
\min _{\Sigma_{H \mathbf{R}}^{\prime}}[u(z)-u(-\bar{z})]=\min _{\Sigma_{H \mathbf{R}}^{\prime \prime}}[u(z)-u(-\bar{z})]=2(\operatorname{Re} z)\left(1-\frac{1}{|z|^{2}}\right) .
$$

For each $z$, the maxima in (a) are achieved if and only if $f(z)=z-1 / \bar{z}+$ $2 \arg ((1+i / z) /(1-i / z))$. In (b) the first minimum is achieved if and only if $f(z)=z-1 / \bar{z}+A \log |z|$ with $-2 \leq A \leq 2$ and the second one if and only if $f(z)=z-1 / \bar{z}$.

ProOF. Since

$$
u(z)-u(-\bar{z})=2(\operatorname{Re} z)\left(1-\frac{1}{|z|^{2}}\right)-\int_{|\eta|=1} 2 \operatorname{Im}\{2 \eta+A\} \arg \left(\frac{1-\eta / z}{1+\eta / \bar{z}}\right) d \mu
$$

for $f=u+i v \in \Sigma_{H \mathbf{R}}^{\prime}$, we have to find the maximum and minimum of

$$
I=-4 \int_{0}^{2 \pi}(\sin \theta-\sin \beta) \arg \left(\frac{1-e^{i \theta} / z}{1+e^{i \theta} / \bar{z}}\right) d \tilde{\mu}
$$

where $\sin \beta=\int_{0}^{2 \pi} \sin \theta d \tilde{\mu}$, over probability measures $\tilde{\mu}$ on $[0,2 \pi]$. If $w=$ $2 i z /\left(1-z^{2}\right)$, then $\operatorname{Im} w<0$ and

$$
\arg \left(\frac{1-e^{i \theta} / z}{1+e^{i \theta} / \bar{z}}\right)=\arg \left(1-\frac{1}{z^{2}}-\frac{2 i \sin \theta}{z}\right)=\arg \left(1-\frac{1}{z^{2}}\right)+\arg (1+w \sin \theta)
$$

up to an integer mulitple of $2 \pi$. Therefore

$$
\begin{aligned}
I & =-4 \int_{0}^{2 \pi}(\sin \theta-\sin \beta) \arg (1+w \sin \theta) d \tilde{\mu} \\
& =-4 \int_{-1}^{1}\left(x-x_{0}\right) \arg (1+x w) d \hat{\mu},
\end{aligned}
$$

where $x_{0}=\int_{-1}^{1} x d \hat{\mu}$ and $\hat{\mu}$ is a probability measure on $[-1,1]$.

It is convenient to write

$$
I=4 \int_{-1}^{1}\left(x-x_{0}\right) \arg \left(\frac{1+x_{0} w}{1+x w}\right) d \hat{\mu}
$$

since the function $G(x)=\left(x-x_{0}\right) \arg \left(\left(1+x_{0} w\right) /(1+x w)\right)$ is nonnegative on $[-1,1]$. Therefore $I \geq 0$ and $u(z)-u(-\bar{z}) \geq 2(\operatorname{Re} z)\left(1-1 /|z|^{2}\right)$. Equality is possible if and only if $\hat{\mu}$ is concentrated where $G$ vanishes, that is, at $x=x_{0}$. Since 
the corresponding measure $\mu$ on $|\eta|=1$ is concentrated where $\operatorname{Im} \eta$ is constant, the extremal functions in $\Sigma_{H \mathbf{R}}^{\prime}$ are the ones given. Since the extremal function $f(z)=z-1 / \bar{z}$ belongs also to $\Sigma_{H \mathbf{R}}^{\prime \prime}$, the minimum problem for $\Sigma_{H \mathbf{R}}^{\prime \prime}$ follows from that for $\Sigma_{H \mathbf{R}}^{\prime}$.

To find the maximum of $I$, observe that $G$ is an increasing function of $\left|x-x_{0}\right|$. Therefore, $I$ will be a maximum only when $\hat{\mu}$ is concentrated at $x= \pm 1$. If $\lambda=\hat{\mu}(1)$, then $x_{0}=2 \lambda-1$ and from (4.9),

$$
\begin{aligned}
I & =-4[\lambda(2-2 \lambda) \arg (1+w)+(1-\lambda)(-2 \lambda) \arg (1-w)] \\
& =8 \lambda(1-\lambda) \arg \left(\frac{1-w}{1+w}\right) \leq 2 \arg \left(\frac{1-w}{1+w}\right)=4 \arg \left(\frac{1+i / z}{1-i / z}\right) .
\end{aligned}
$$

Equality occurs if and only if $\hat{\mu}$ has mass $\lambda=1 / 2$ at $x=1$ and mass $1-\lambda=\frac{1}{2}$ at $x=-1$, which corresponds to the indicated extremal function.

Our final applications concern coefficient problems for $\Sigma_{H \mathbf{R}}^{\prime}$ and $\Sigma_{H \mathbf{R}}^{\prime \prime}$. We have already seen in Theorem 3.2 that for

$$
f(z)=z+A \log |z|+\sum_{n=1}^{\infty} a_{n} z^{-n}+\overline{\sum_{n=1}^{\infty} b_{n} z^{-n}}
$$

we have

$$
\begin{aligned}
& \max _{\Sigma_{H}^{\prime}}|A|=\max _{\Sigma_{H \mathbf{R}}^{\prime}}|A|=2, \\
& \max _{\Sigma_{H}^{\prime}}\left|b_{1}\right|=\max _{\Sigma_{H}^{\prime \prime}}\left|b_{1}\right|=\max _{\Sigma_{H \mathbf{R}}^{\prime}}\left|b_{1}\right|=\max _{\Sigma_{H \mathbf{R}}^{\prime \prime}}\left|b_{1}\right|=1 .
\end{aligned}
$$

Indeed, the functions $f(z)=z-1 / \bar{z}+A \log |z|$ belong to $\Sigma_{H \mathbf{R}}^{\prime}$ for $|A| \leq 2$ and to $\Sigma_{H \mathbf{R}}^{\prime \prime}$ for $A=0$.

From (4.1) we obtain the representations

$$
\begin{aligned}
& a_{n}=\frac{-i}{n} \int_{|\eta|=1} \eta^{n} \operatorname{Im}\{2 \eta+A\} d \mu \quad \text { for } n \geq 1 \\
& a_{1}=b_{1}+1, \quad \text { and } \\
& a_{n}=b_{n} \quad \text { for } n \geq 2
\end{aligned}
$$

More convenient will be the representation

$$
a_{n}=\frac{-2 i}{n} \int_{0}^{2 \pi} e^{i n \theta}(\sin \theta-\sin \beta) d \mu \quad \text { for } n \geq 1,
$$

where $\sin \beta=\int_{0}^{2 \pi} \sin \theta d \mu$ and $\mu$ is a probability measure on $[0,2 \pi]$.

THEOREM 4.7. If $f \in \Sigma_{H \mathbf{R}}^{\prime}$, then $\left|a_{1}\right| \leq 2$ and for all $n \geq 2$,

$$
\left|a_{n}\right|=\left|b_{n}\right| \leq 2 / n \text {. }
$$

For odd indices the inequalities are sharp, even for $\Sigma_{H \mathbf{R}}^{\prime \prime}$, and equality occurs if and only if $f(z)=z-1 / \bar{z}+2 \arg ((1+i / z) /(1-i / z))$.

PROOF. Using (4.10), we have

$$
\begin{aligned}
\left|\frac{n}{2} a_{n}\right|^{2} & \leq\left(\int_{0}^{2 \pi}|\sin \theta-\sin \beta| d \mu\right)^{2} \leq \int_{0}^{2 \pi}(\sin \theta-\sin \beta)^{2} d \mu \\
& =\int_{0}^{2 \pi} \sin ^{2} \theta d \mu-\sin ^{2} \beta \leq 1
\end{aligned}
$$


Equality could occur only if $\sin \beta=0$ and $\mu$ were concentrated where $\sin ^{2} \theta=1$, that is, for the function given. Equality actually does occur for the odd coefficients, but not for the even ones.

Since the extremal functions have real coefficients and belong to $\Sigma_{H \mathbf{R}}^{\prime \prime}$, the following is obvious.

COROLLARY 4.8. For the odd coefficients we have

$$
\max _{\Sigma_{H \mathbf{R}}^{\prime}}\left|\operatorname{Re} a_{1}\right|=\max _{\Sigma_{H \mathbf{R}}^{\prime \prime}}\left|\operatorname{Re} a_{1}\right|=2, \quad \max _{\Sigma_{H \mathbf{R}}^{\prime}}\left|\operatorname{Re} b_{1}\right|=\max _{\Sigma_{H \mathbf{R}}^{\prime \prime}}\left|\operatorname{Re} b_{1}\right|=1,
$$

and

$$
\max _{\Sigma_{H \mathbf{R}}^{\prime}}\left|\operatorname{Re} a_{2 n+1}\right|=\max _{\Sigma_{H \mathbf{R}}^{\prime \prime}}\left|\operatorname{Re} a_{2 n+1}\right|=\max _{\Sigma_{H \mathbf{R}}^{\prime}}\left|\operatorname{Re} b_{2 n+1}\right|=\max _{\Sigma_{H \mathbf{R}}^{\prime \prime}}\left|\operatorname{Re} b_{2 n+1}\right|=\frac{2}{2 n+1}
$$

for $n=1,2,3, \ldots$

Let us consider the even coefficients for $\Sigma_{H \mathbf{R}}^{\prime}$. The estimates given in Theorem 4.7 are not sharp. To simplify the development we shall consider only their real parts. The answers will be in terms of the Chebyshev polynomials of second kind defined by

or

$$
U_{n}(\cos \theta)=\frac{\sin [(n+1) \theta]}{\sin \theta}
$$

$$
U_{n}(x)=\sum_{k=0}^{[n / 2]} \frac{(-1)^{k}(n-k) !}{k !(n-2 k) !}(2 x)^{n-2 k} .
$$

THEOREM 4.9. For all even coefficients we have

$$
\max _{\Sigma_{H \mathbf{R}}^{\prime}}\left|\operatorname{Re} a_{2 n}\right|=\max _{\Sigma_{H \mathbf{R}}^{\prime}}\left|\operatorname{Re} b_{2 n}\right|=\frac{1}{n} \max _{-1 \leq x \leq 1}\left(1-x^{2}\right) U_{2 n-1}(x) .
$$

ProOF. Applying the Cauchy-Schwarz inequality to (4.10), we obtain

$$
\begin{aligned}
\left(n \operatorname{Re} a_{2 n}\right)^{2} & \leq\left(\int_{0}^{2 \pi} \sin ^{2}(2 n \theta) d \mu\right)\left(\int_{0}^{2 \pi} \sin ^{2} \theta d \mu-\sin ^{2} \beta\right) \\
& \leq \int_{0}^{2 \pi} \sin ^{2}(2 n \theta) d \mu \cdot \int_{0}^{2 \pi} \sin ^{2} \theta d \mu .
\end{aligned}
$$

From the periodicity of $\sin ^{2}(2 n \theta)$ and $\sin ^{2} \theta$ and the monotonicity of $\sin ^{2} \theta$ on $[0, \pi / 2]$, it is sufficient to estimate

$$
\int_{\hat{\theta}_{n}}^{\pi / 2} \sin ^{2}(2 n \theta) d \mu \cdot \int_{\hat{\theta}_{n}}^{\pi / 2} \sin ^{2} \theta d \mu
$$

where $\hat{\theta}_{n}=\pi / 2-\pi /(2 n)$, over probability measures on $\left[\hat{\theta}_{n}, \pi / 2\right]$.

Consider therefore the Lagrangian

$$
L=\sum_{j=1}^{N} \lambda_{j} \sin ^{2}\left(2 n \theta_{j}\right) \sum_{k=1}^{N} \lambda_{k} \sin ^{2} \theta_{k}-B\left(\sum_{k=1}^{N} \lambda_{k}-1\right)
$$


where $\lambda_{k}>0$ and $\theta_{k} \in\left[\hat{\theta}_{n}, \pi / 2\right]$. Differentiation with respect to $\lambda_{k}$ gives

$$
\frac{\partial L}{\partial \lambda_{k}}=\sin ^{2} \theta_{k} \sum_{j=1}^{N} \lambda_{j} \sin ^{2}\left(2 n \theta_{j}\right)+\sin ^{2}\left(2 n \theta_{k}\right) \sum_{j=1}^{N} \lambda_{j} \sin ^{2} \theta_{j}-B=0 .
$$

The maximum $M$ of our functional is clearly positive, and

$$
\sum_{k=1}^{N} \lambda_{k} \frac{\partial L}{\partial \lambda_{k}}=2 M-B=0 \quad \text { or } \quad B=2 M>0 .
$$

We now show that optimal measures have no mass at the endpoints. Indeed, if $\theta_{k}$ were $\hat{\theta}_{n}$ or $\pi / 2$, then $\sin ^{2}\left(2 n \theta_{k}\right)=0$ and (4.11) would imply

$$
0<2 M=A=\sin ^{2} \theta_{k} \sum_{j=1}^{N} \lambda_{j} \sin ^{2}\left(2 n \theta_{j}\right)<1
$$

or $M<\frac{1}{2}$. On the other hand, the measure with unit mass at $\pi / 2-\pi /(4 n)$ shows that $M \geq \cos ^{2}(\pi /(4 n)) \geq \cos ^{2}(\pi / 4)=1 / 2$.

Differentiation of $L$ with respect to $\theta_{k}$ gives

$$
\frac{1}{\lambda_{k}} \frac{\partial L}{\partial \lambda_{k}}=\sin \left(2 \theta_{k}\right) \sum_{j=1}^{N} \lambda_{j} \sin ^{2}\left(2 n \theta_{j}\right)+\sin \left(4 n \theta_{k}\right) \sum_{j=1}^{N} \lambda_{j} \sin ^{2} \theta_{j}=0 .
$$

That is, $\sin \left(4 n \theta_{k}\right) / \sin \left(2 \theta_{k}\right)$ is a negative constant for all $k$. The function $\sin (4 n \theta) / \sin (2 \theta)$ is positive on the interval $\left(\hat{\theta}_{n}, \pi / 2-\pi /(4 n)\right)$ and negative on $(\pi / 2-\pi /(4 n), \pi / 2)$. Furthermore, on $(\pi / 2-\pi /(4 n), \pi / 2)$ it is strictly decreasing. Therefore, there is only one point $\tilde{\theta}_{n}$ satisfying (4.12), and it lies in the interval $(\pi / 2-\pi /(4 n), \pi / 2)$. As a result, we have

$$
\left(n \operatorname{Re} a_{2 n}\right)^{2} \leq \sin ^{2}\left(2 n \tilde{\theta}_{n}\right) \sin ^{2} \tilde{\theta}_{n} .
$$

The function $f \in \Sigma_{H \mathbf{R}}^{\prime}$ whose measure consists of equal point masses at $\eta=e^{i \tilde{\theta}_{n}}$ and $\eta=e^{-i \tilde{\theta}_{n}}$ has coefficient $a_{2 n}$ that achieves equality in (4.13). Therefore we have

$$
\max _{\Sigma_{H \mathbf{R}}^{\prime}}\left|\operatorname{Re} a_{2 n}\right|=\frac{1}{n} \max _{0 \leq \theta \leq 2 \pi}|\sin (2 n \theta) \cdot \sin \theta|=\frac{1}{n} \max _{-1 \leq x \leq 1}\left|\left(1-x^{2}\right) U_{2 n-1}(x)\right| .
$$

Furthermore, since $U_{2 n-1}$ is odd, we may omit its absolute value sign.

We now apply Theorem 4.9 to the second and fourth coefficients. One easily verifies that

$$
\begin{aligned}
& \max _{-1 \leq x \leq 1}\left(1-x^{2}\right) U_{1}(x)=\max _{-1 \leq x \leq 1} 2 x\left(1-x^{2}\right)=\frac{4}{9} \sqrt{3}=.76980 \ldots \\
& \max _{-1 \leq x \leq 1} \frac{1}{2}\left(1-x^{2}\right) U_{3}(x)=\max _{-1 \leq x \leq 1} 2 x\left(2 x^{2}-1\right)\left(1-x^{2}\right) \\
&=\frac{\sqrt{411+41 \sqrt{41}}}{25 \sqrt{5}}=.46425 \ldots
\end{aligned}
$$


COROLLARY 4.10. We have $\max _{\Sigma_{H \mathrm{R}}^{\prime}}\left|\operatorname{Re} a_{2}\right|=\max _{\Sigma_{\Sigma_{\mathrm{R}}}^{\prime}}\left|\operatorname{Re} b_{2}\right|=\frac{4}{9} \sqrt{3}$ and $\max _{\Sigma_{H \mathrm{R}}^{\prime}}\left|\operatorname{Re} a_{4}\right|=\max _{\Sigma_{H \mathrm{R}}^{\prime}}\left|\operatorname{Re} b_{4}\right|=\sqrt{411+41 \sqrt{41}} /(25 \sqrt{5})$.

REMARKS. If $f$ belongs to $\Sigma_{H \mathbf{R}}^{\prime}$, then so does $-f(-z)$ and the even coefficients change sign. Therefore we may replace $\left|\operatorname{Re} a_{2 n}\right|$ by $\operatorname{Re} a_{2 n}$ or $-\operatorname{Re} a_{2 n}$ in Theorem 4.9 and Corollary 4.10. A similar statement holds for $b_{2 n}$.

If $\theta_{n}^{*}=\pi / 2+(-1)^{n} \pi /(4 n)$, then $\left(\sin ^{2} \theta_{n}^{*}\right) U_{2 n-1}\left(\cos \theta_{n}^{*}\right)=\cos (\pi /(4 n))$ approaches one as $n \rightarrow \infty$. As a result, $\max _{-1 \leq x \leq 1}\left(1-x^{2}\right) U_{2 n-1}(x)$ approaches one as $n \rightarrow \infty$. Thus the bounds in Theorem 4.7 are asymptotically correct for the even coefficients.

COROLlary 4.11. We have

$$
\lim _{n \rightarrow \infty} \max _{\Sigma_{H \mathbf{R}}^{\prime}} n \operatorname{Re} a_{2 n}=\lim _{n \rightarrow \infty} \max _{\Sigma_{\mathbf{R}}^{\prime}} n \operatorname{Re} b_{2 n}=1 .
$$

In $\Sigma_{H \mathbf{R}}^{\prime \prime}$, Theorem 4.7 gave sharp estimates for the odd coefficients. We shall briefly consider the even coefficients.

The function

$$
f(z)=z-\frac{1}{\bar{z}}-\frac{2}{\sqrt{3}} \arg \left(\frac{1-e^{\pi i / 3} / z}{1-e^{-\pi i / 3} / z}\right)
$$

belongs to $\Sigma_{H \mathbf{R}}^{\prime \prime}$. It arises from the measure with mass $\frac{1}{3}$ at the cube roots of -1 . Its coefficients $a_{2}=b_{2}=\frac{1}{2}$. Thus the bound $\left|b_{2}\right| \leq \frac{1}{2}$ in $\Sigma_{H}^{\prime \prime}$ from Theorem 3.2 is sharp also in $\Sigma_{H \mathbf{R}}^{\prime \prime}$.

THEOREM 4.12. We have $\max _{\Sigma_{H \mathbf{R}}^{\prime \prime}} \operatorname{Re} a_{2}=\max _{\Sigma_{H \mathbf{R}}^{\prime \prime}} \operatorname{Re} b_{2}=\max _{\Sigma_{H \mathbf{R}}^{\prime \prime}}\left|a_{2}\right|=$ $\max _{\Sigma_{H \mathbf{R}}^{\prime \prime}}\left|b_{2}\right|=\frac{1}{2}$.

The problem for higher even coefficients in $\Sigma_{H \mathbf{R}}^{\prime \prime}$ is more difficult. We may write the coefficient

$$
n \operatorname{Re} a_{2 n}=\int_{0}^{2 \pi} \sin (2 n \theta) \sin \theta d \mu=\int_{-1}^{1}\left(1-x^{2}\right) U_{2 n-1}(x) d \tilde{\mu}
$$

for some probability measure $\tilde{\mu}$ on $[-1,1]$. Since the integrand is a function only of $x=\cos \theta$, the only new constraint is $\int_{-1}^{1} x d \tilde{\mu}=0$. Thus the Lagrangian to be optimized becomes

$$
L=\frac{1}{n} \sum_{k=1}^{N} \lambda_{k}\left(1-x_{k}^{2}\right) U_{2 n-1}\left(x_{k}\right)-B\left(\sum_{k=1}^{N} \lambda_{k}-1\right)-C \sum_{k=1}^{N} \lambda_{k} x_{k} .
$$

Using this technique, one obtains, for example,

$$
\max _{\Sigma_{H \mathbf{R}}^{\prime \prime}} \operatorname{Re} a_{4}=\max _{\Sigma_{H \mathbf{R}}^{\prime \prime}} \operatorname{Re} b_{4}=\frac{18}{25} \sqrt{\frac{3}{10}}=.39436 \ldots
$$

The optimal measure has mass $(5-\sqrt{5}) / 20$ at the points $\pm e^{i \theta_{1}}$, where $\cos \theta_{1}=$ $\sqrt{3 / 40}(1+\sqrt{5})$, and mass $(5+\sqrt{5}) / 20$ at the points $\pm e^{i \theta_{2}}$, where $\cos \theta_{2}=$ $\sqrt{3 / 40}(1-\sqrt{5})$. 


\section{REFERENCES}

1. G. Choquet, Sur un type de transformation analytique généralisant la représentation conforme et définie au moyen de fonctions harmoniques, Bull. Sci. Math. 69 (1945), 156-165.

2. J. Clunie and T. Sheil-Small, Harmonic univalent functions, Ann. Acad. Sci. Fenn. Ser. A I Math. 9 (1984), 3-25.

3. R. R. Hall, On an inequality of E. Heinz, J. Analyse Math. 42 (1983), 185-198.

4. E. Heinz, Über die Lösungen der Minimalflächengleichung, Nachr. Akad. Wiss. Göttingen, Math.-Phys. Kl. (1952), 51-56.

5. W. Hengartner and G. Schober, On schlicht mappings to domains convex in one direction, Comment. Math. Helv. 45 (1970), 303-314.

6. O. Lehto and K. I. Virtanen, Quasiconformal mappings in the plane, Springer-Verlag, Berlin-Heidelberg-New York, 1973.

7. K. Löwner, Untersuchungen über schlichte konforme Abbildungen des Einheitskreises. I, Math. Ann. 89 (1923), 103-121.

8. D. J. Hallenbeck and T. H. MacGregor, Support points of families of analytic functions described by subordination, Trans. Amer. Math. Soc. 278 (1983), 523-546.

DÉPartement de Mathématiques, Université LaVal, QUÉbec, P.Q. G1K 7P4, CANADA

DEPARTMENT OF MAThEMATICS, INDiANA UNIVERSity, BloOMington, INDiANA 47405 